\title{
L'ESPANSIONE DEL POTERE GIUDIZIARIO NELLE DEMOCRAZIE CONTEMPORANEE
}

\author{
di Carlo Guarnieri e Patrizia Pederzoli
}

In una recente rassegna dei rapporti fra magistratura e politica - dal significativo titolo «L'espansione globale del potere giudiziario» (Tate e Vallinder 1995) - si è giunti parlare di un processo di «giudiziarizzazione della politica» che interessa ormai tutti i regimi democratici, e cioè di un' «espansione del raggio d'azione dei tribunali o dei giudici a spese dei politici e/o degli amministratori: in altre parole lo spostamento dei diritti di decisione dal legislativo, il governo o l'amministrazione verso $i$ tribunali» (Vallinder 1995, 13). Le ragioni di questo fenomeno sono numerose e vanno collegate a tendenze di lungo periodo che si manifestano, ma con maggior o minor forza, in tutti i regimi democratici (Pederzoli 1990). In realtà, non si tratta di un fenomeno del tutto nuovo in paesi come gli Stati Uniti, ma di recente si è manifestato anche nelle democrazie dell'Europa latina: Spagna, Francia e soprattutto Italia. Per comprendere le ragioni alla base di questa nuova rilevanza dell'azione giudiziaria analizzeremo questi tre casi insieme a Stati Uniti - un sistema politico da sempre contraddistinto da un notevole attivismo giudiziario - Inghilterra e Germania, due paesi che, pur appartenendo a due diverse tradizioni giuridiche - rispettivamente di common e civil law - sono stati caratterizzati, almeno fino ad oggi, da una minore propensione della magistratura a intervenire nel processo politico ${ }^{1}$.

In realtà, gli elementi da considerare per analizzare il pro-

L'articolo è una sintesi del secondo capitolo, Il sistema giudiziario, del volume Le democrazie giudiziarie. Potere giudiziario e democrazia, di prossima pubblicazione presso Il Mulino cui si invia per l'analisi dettagliata dei singoli casi. Il paragrafo sul pubblico ministero è stato redatto da Carlo Guarnieri, gli altri da Patrizia Pederzoli.

1 Per certi aspetti, terremo conto anche dell'esperienza del Portogallo.

RIVISTA ITALIANA DI SCIENZA POLITICA / a. XXVI, n. 2, agosto 1996 
cesso di «giudiziarizzazione della politica» sono diversi: i giudici in primo luogo, e quindi le modalità con cui vengono reclutati e il loro status così come la concezione di ruolo cui tendono ad aderire; l'assetto del sistema giudiziario e, infine, i caratteri del sistema politico in cui la magistratura opera (Guarnieri e Pederzoli 1996). In questo articolo ci concentreremo sul sistema giudiziario ed in particolare sulla sua articolazione strutturale, sulle modalità con cui può essere attivato e sui poteri che vengono attribuiti al giudice. L'obiettivo è di costruire in questo modo uno schema d'analisi in grado di indicare quelle configurazioni del sistema giudiziario maggiormente atte, a parità di altri elementi, a favorire in un regime democratico la crescita del potere della magistratura.

\section{La struttura del sistema giudiziario}

I sistemi giudiziari possono essere visti come organizzazioni complesse, la cui struttura tende in parte a riflettere il carattere via via più composito e diversificato del contenzioso che devono trattare. Un primo elemento di complessità deriva dal loro essere generalmente molto articolati sul territorio, fattore che dà luogo ad una frammentazione più o meno elevata dei centri decisionali, ossia dei singoli organi giudicanti. Quello della «giustizia vicina al cittadino» è infatti un principio ormai largamente accettato in molti paesi, anche se si traduce in soluzioni diverse sul piano organizzativo e ordinamentale, che vanno dal giudice di pace italiano sino alle small claims courts inglesi. L'esigenza di fornire risposte modellate sui caratteri specifici delle controversie di volta in volta demandate al giudice costituisce un secondo elemento suscettibile di accrescere la differenziazione interna dei sistemi giudiziari. Ne costituisce un esempio la presenza in molti paesi di corti specializzate nel trattare alcuni tipi di dispute, che possono andare dal contenzioso minorile sino alle questioni in materia di immigrazione. Infine, la complessità dei sistemi giudiziari si collega anche ad una garanzia che tutti gli ordinamenti riconoscono ai cittadini coinvolti in un processo: il doppio grado di giudizio. La parte soccombente ha cioè diritto ad una seconda possibilità innanzi ad un giudice diverso da quello che ha deciso la causa in prima istanza. In questo modo la dimensione verticale del sistema, ossia l'ordine sostanzialmente gerarchico fra i vari gradi di giudizio, 
interseca quella orizzontale, rappresentata dalla sua diffusione sul territorio (Shapiro 1980). L'immagine che meglio descrive questa struttura è in effetti quella di una piramide che si sviluppa su tre livelli: una base di solito molto estesa e formata da un numero variabile di corti a competenza territorialmente limitata; un livello intermedio che, pur dotato di una competenza originaria, è soprattutto chiamato a decidere degli appelli contro le sentenze rese dalle corti inferiori; infine un vertice a giurisdizione nazionale, il cui compito precipuo consiste nel garantire la coerenza dell'interpretazione giurisprudenziale del diritto così da bilanciare la pluralità delle corti di merito - ma che di fatto opera spesso come vero e proprio terzo grado di giudizio.

$\mathrm{Al}$ di là di questo profilo generale, che può essere facilmente identificato in tutti i paesi, la struttura del sistema giudiziario presenta numerose differenze significative passando da un caso all'altro. Concentreremo qui la nostra attenzione sulle sole variazioni che sembrano influire, in modo diretto o indiretto, sulla rilevanza politica della giustizia, prendendone in esame due in particolare. La prima è data da ciò che possiamo definire come il grado di coesione ovvero di frammentazione delle competenze giurisdizionali. Detto altrimenti, si tratta di considerare quali sono le controversie che il giudice può essere chiamato a decidere, un aspetto questo che consente di valutarne il raggio d'intervento potenziale, che del resto non coincide necessariamente con quello effettivo. L'eventualità che il giudice sia concretamente attivato dipende infatti da un insieme di condizioni che vedremo più da vicino nel prossimo paragrafo. Per il momento, ci limiteremo a considerare se la competenza a conoscere dei diversi tipi di controversie è concentrata in una sola giurisdizione o se è per contro ripartita fra una pluralità di giurisdizioni diverse, che diano luogo ad altrettante piramidi organizzative, separate ed autonome l'una dall'altra. È evidente che, ceteris paribus, quanto più ampia è la sfera decisionale del giudice tanto più rilevante e politicamente incisivo potrà essere il suo ruolo. Infatti, al di là degli obiettivi formalmente enunciati, l'istituzione di corti speciali tende a risolversi nella parallela contrazione delle competenze assegnate al giudice ordinario relativamente a materie che di solito chiamano in causa interessi di ampia portata e, dunque, di natura esplicitamente politica. Non è un caso del resto che tali corti siano per lo più formate da giudici appartenenti ad un corpo diverso, separato dalla magistratura ordinaria e con garanzie di indipendenza più attenuate rispetto a 
questa, per effetto soprattutto di un più stretto collegamento con il sistema politico e particolarmente con l'esecutivo (Merryman 1985).

L'importanza della frammentazione giurisdizionale, come strumento che consente di sterilizzare politicamente la giustizia ordinaria e di preservarne nel contempo una certa autonomia, è ben documentata dalle strategie di controllo attuate dai regimi autoritari. In Italia, l'assetto burocratico della magistratura non viene radicalmente modificato dal regime fascista. Mantenendo il reclutamento per concorso, questi rinuncia ad intervenire direttamente sulla composizione del corpo giudiziario per conquistarne la lealtà, tanto più che gli orientamenti condivisi dalla magistratura e il prevalere di una concezione di ruolo chiaramente esecutoria non sembrano costituire una minaccia in questo senso. Benché l'assetto gerarchico dell'organizzazione giudiziaria costituisca in sé uno strumento di controllo da non sottovalutare, ma che ad ogni modo era già presente in epoca liberale, l'obiettivo perseguito dal regime, prima della virata in direzione più chiaramente totalitaria alla fine degli anni ' 30 , consiste piuttosto nel depoliticizzare la magistratura. L'istituzione di tribunali speciali, con competenze relative all'ordine pubblico e dunque soprattutto in materia penale, può essere considerata parte integrante di questo progetto, in quanto consente di non coinvolgere la magistratura nella repressione dei reati politici. Una situazione per molti versi analoga a questa può essere riscontrata nella Spagna franchista, anche se con sviluppi che seguono un andamento inverso a quello italiano. Le tendenze più chiaramente totalitarie, che si manifestano all'atto di instaurazione del regime e che vedono il coinvolgimento diretto delle corti nella repressione politica, si allentano nel periodo successivo. A partire dagli anni '50 il regime presenta così alcuni dei tratti tipici dell'autoritarismo, soprattutto per quanto attiene alle strategie di controllo sull'amministrazione della giustizia. Anche in questo caso il corpo giudiziario conserva il tradizionale assetto burocratico e tanto il reclutamento quanto i meccanismi di progressione in carriera restano in gran parte sottratti alle interferenze esterne. La magistratura spagnola denota così, se non un'autentica indipendenza istituzionale, quanto meno una certa autonomia. Una constatazione questa che non deve sorprendere. Tale autonomia relativa può essere infatti spiegata proprio con la creazione di numerose giurisdizioni speciali. Formati da giudici nominati direttamente dal regime e che questi 
può in qualsiasi momento trasferire o destituire, i tribunali speciali sono investiti di tutti i casi politicamente sensibili. Di converso, la magistratura ordinaria vede il proprio raggio d'azione circoscritto essenzialmente al contenzioso privato, ossia a dispute ininfluenti rispetto agli obiettivi di continuità di un regime fondato sull'acquiescenza piuttosto che sulla mobilitazione (Toharia 1975). La mancanza di rilievo politico, dovuta al tipo di casi su cui è chiamata a decidere, può dunque essere considerato il pedaggio che la magistratura spagnola corrisponde alla relativa autonomia di cui gode ${ }^{2}$.

L'interesse per l'ampiezza della giurisdizione, pur essendo suggerito dalle esperienze autoritarie, si giustifica anche rispetto ai regimi democratici, particolarmente quelli dell'Europa continentale che sono tuttora caratterizzati dalla segmentazione delle strutture giurisdizionali. A questo punto, e prima di procedere, è però possibile delineare due diversi tipi di strumenti, che d'altronde non si escludono a vicenda, con cui il sistema politico può influire sull'amministrazione della giustizia (Guarnieri e Pederzoli 1996, cap. 2). Un primo canale d'influenza sul giudiziario tocca direttamente lo status dei magistrati e passa o attraverso le modalità di reclutamento o attraverso i meccanismi di progressione in carriera, una distinzione questa che ricalca sostanzialmente le differenze riscontrabili tra magistrature professionali, tipiche dei paesi di common law, e burocratiche, prevalenti in quelli di civil law. Una seconda e più indiretta strategia di controllo attiene invece alla struttura della giurisdizione e mira a ridurre l'impatto potenziale del giudiziario, limitandone l'area di intervento, così da costringerlo ad un ruolo di basso profilo politico. In effetti, è possibile valutare il rilievo politico del giudice in base al tipo di controversie su cui è chiamato a decidere. Più precisamente, questo criterio d'analisi ha riguardo all'identità pubblica o privata delle controparti in conflitto, che costituisce un indicatore attendibile circa la natura e la portata degli interessi in gioco nella disputa. Seguendo questa prospettiva, possiamo allora distinguere: le controversie fra privati cittadini, la cui implicazioni politiche sono generalmente, ma non necessariamente, basse; le controversie che invece vedono come parte in causa un soggetto pubblico, come nel contenzioso pe-

2 Anche il regime portoghese di Caetano e Salazar mostra un andamento simile a quello spagnolo. 
nale o amministrativo; infine, i conflitti fra poteri dello stato, o ancora fra governo centrale e locale (Friedman 1976). Come vedremo fra poco, mentre il contenzioso penale è ormai ovunque affidato alla giurisdizione ordinaria, con la sola eccezione di quello militare, le controversie di diritto amministrativo e costituzionale sono spesso attribuite, soprattutto in Europa, a corti istituite ad boc. A fianco di questo tipo di frammentazione orizzontale è comunque possibile osservare un'ulteriore forma di divisione delle competenze, che risponde però ad una logica diversa, anche se egualmente suscettibile di ridurre il raggio d'azione del giudice. Il dualismo delle strutture di governo proprio dei sistemi federali si riflette infatti sulla struttura del giudiziario, dando luogo ad una partizione fra corti federali e statali, che ha per effetto quello di comprimere le rispettive aree di intervento potenziale.

Il secondo elemento di variazione concerne invece i rapporti che si stabiliscono tra i diversi livelli che formano la piramide giurisdizionale. Le differenze più importanti in questo senso non riguardano tanto l'articolazione della struttura, ma piuttosto le sue dinamiche interne e soprattutto il ruolo effettivamente giocato dalle corti supreme, nella loro veste di tutori della coerenza giurisprudenziale. Sotto questo profilo è possibile isolare due modelli alternativi: i sistemi «coordinati», generalmente associati a magistrature professionali, nei quali i ricorsi che giungono al vertice della piramide organizzativa tendono a rappresentare l'eccezione più che la regola, con la conseguenza che buona parte del lavoro decisionale viene di fatto assolto in prima ed ultima istanza dalle corti inferiori; e i sistemi «gerarchici», per lo più associati a magistrature di impianto burocratico, i cui percorsi di carriera ricalcano la struttura stessa della piramide, nei quali una porzione molto consistente del lavoro svolto alla base viene riesaminata dalle corti supreme, che assumono in tal modo un'autentica funzione di controllo sugli indirizzi giurisprudenziali promossi dalle corti inferiori (Damaska 1975 e 1991; Shapiro 1980). Questi modelli sono dunque mossi da logiche diverse. Per la parte che qui interessa, tali differenze sono riconducibili al ruolo di volta in volta esercitato dalle corti supreme, ai meccanismi istituzionali suscettibili di rafforzarne o meno l'autorevolezza, nonché alle norme che concorrono a governare il flusso dei casi trattati, come ad esempio quelle che disciplinano i mezzi di impugnazione delle sentenze. In linea di principio le strutture caratterizzate da una dinamica centripeta, 
ossia dalla presenza di un vertice decisionale forte, appaiono meglio equipaggiate per assicurare la coerenza giurisprudenziale - considerata in questo caso un valore prioritario e funzionale alla certezza del diritto - ciò che può a propria volta accrescere l'incidenza politica del sistema giudiziario. In effetti, politiche giurisprudenziali relativamente stabili e soprattutto coerenti possono costituire un referente non solo per i singoli, ma anche per i gruppi d'interesse. Di converso, una dinamica centrifuga, che incoraggi cioè l'autonomia dei singoli centri decisionali dislocati lungo l'intera piramide, può ridurre sensibilmente il grado di coerenza giurisprudenziale - che è in questo caso considerata meno importante, anche in ragione di un approccio più pragmatico al principio di certezza del diritto - e alimentare contrasti interni tali da ridurre, almeno in linea di principio, l'impatto politico del sistema. D'altra parte, è però evidente che una giurisprudenza «a più voci» può sì minare la coerenza del sistema ed alimentare in certa misura la litigiosità (Toharia 1976; Tarello 1988), ma tende a rafforzare l'immagine d'imparzialità del giudice, che appare in questo caso meno influenzato dagli orientamenti avallati dal vertice decisionale (Eckhoff 1965, 39). Infine, non va dimenticata la possibilità che un minor grado di coerenza interna - in altre parole la compresenza di indirizzi giurisprudenziali difformi - possa aumentare le occasioni di ricorso al giudice, che può in tal modo divenire un interlocutore ricercato dal suo ambiente politico e sociale.

L'aspetto dei sistemi giudiziari di common law che più colpisce l'attenzione di un osservatore continentale è dato probabilmente dalla loro struttura a giurisdizione unica. Le competenze demandate al giudice si estendono dalle controversie civili e penali sino a quelle di diritto pubblico e, nel caso degli Stati Uniti, al contenzioso costituzionale.

$\mathrm{La}$ frammentazione giurisdizionale è invece un dato che caratterizza in genere $i$ sistemi continentali. Con la sola eccezione della Spagna, in tutti gli altri casi considerati è possibile riscontrare una partizione fondamentale, ereditata dal modello napoleonico, tra la giurisdizione ordinaria, a competenza civile e penale, e quella amministrativa cui sono demandate le controversie che vedono un'amministrazione pubblica quale parte in causa (Waltman e Holland 1988). Tenendo conto delle dimensioni raggiunte dall'intervento pubblico con lo sviluppo dello stato sociale, è evidente che il contenzioso in tal modo sottratto al giudice ordinario deve la sua importanza non solo all'entità de- 
gli interessi in gioco, e dunque al rilievo politico delle relative dispute, ma anche alla loro consistenza quantitativa. Benché importante, la partizione che abbiamo appena tracciato non è tuttavia la sola. A prescindere dall'esistenza o meno di altre corti specializzate, la differenza più immediata rispetto al sistema americano riguarda il controllo di costituzionalità, che viene in questo caso demandato ad apposite corti di cui si parlerà in seguito. Nei sistemi continentali coabitano dunque più piramidi giurisdizionali, che formano altrettanti sottosistemi separati ed in gran parte autonomi l'uno dall'altro. Nella maggior parte dei casi il giudice ordinario tende a mantenere una competenza circoscritta al contenzioso civile e penale. Senza dimenticare che quest'ultimo costituisce storicamente il processo pubblico per antonomasia, resta la circostanza che in tal modo il giudice si vede sottratte dispute che sono divenute via via più importanti e talvolta cruciali, ossia parte di quelle in cui la posta in gioco è data dai diritti del singolo nei suoi rapporti con le istituzioni politiche. Ma non si tratta della sola differenza di rilievo rispetto ai sistemi anglosassoni. Tanto le dinamiche interne alla giurisdizione quanto le funzioni di fatto assunte dalle corti supreme danno luogo ad altrettante differenze significative rispetto ai sistemi anglosassoni e che possono essere messe in relazione con l'assetto burocratico delle magistrature continentali. Salvo qualche sporadica eccezione, questi sistemi non pongono vincoli particolari alla possibilità di invocare l'intervento di una corte superiore. I casi decisi in prima istanza possono essere sempre riesaminati da una corte d'appello, competente a decidere anche sul merito. Non essendo assoggettati alle restrizioni che caratterizzano il mondo di common law, anche i ricorsi alle corti supreme non incontrano generalmente ostacoli formali, poiché queste sono tenute a pronunciarsi su tutte le cause iscritte a ruolo. Se dal punto di vista propriamente normativo le corti supreme sono chiamate a decidere delle sole questioni di diritto, senza entrare cioè nel merito dei singoli casi, in pratica esse finiscono col diventare un autentico terzo livello di giudizio, anche perché la linea di confine tra questioni di fatto e di diritto è spesso, a dir poco, sfumata (Taruffo 1991). Il venir meno dei filtri che altrove governano e distribuiscono i flussi interni al sistema fa dunque sì che una porzione molto consistente dell'intera massa contenziosa riesca ad approdare ai vertici dell'organizzazione (Damaska 1991), che può in tal modo riesaminare una parte cospicua dei casi già processati ai livelli inferiori e 
svolgere compiti di supervisione sul lavoro svolto dai giudici di merito. La funzione così assolta dalle corti supreme è d'altronde perfettamente in linea con la loro matrice storica ed organizzativa. Il modello di riferimento è dato infatti dalla cassazione francese, concepita in origine come organo di controllo sui giudici di merito piuttosto che come garanzia fornita al soccombente. La circostanza che le parti si vedano offrire la possibilità di avere un terzo giudizio altro non è se non un corollario della funzione principale di tali corti, quella di essere cioè poste a presidio della coerenza giurisprudenziale ${ }^{3}$. Benché le sentenze delle corti supreme siano vincolanti rispetto al solo caso in esame - la dottrina dello stare decisis non ha mai trovato accoglimento in questi ordinamenti - la loro forza «persuasiva» è sempre stata in realtà superiore a quella formale (Ancel 1934, 300). L'influenza esercitata delle corti supreme sui giudici di merito, pur in assenza di vincoli formali, è infatti intimamente legata all'assetto burocratico delle magistrature continentali e in particolare al ruolo svolto dall'élite giudiziaria, che siede in tali corti, nei meccanismi di avanzamento in carriera (Friedrich 1950). Tuttavia, la loro posizione classica, quali centri decisionali forti del sistema, sembra oggi sensibilmente indebolita. Non è probabilmente un caso che questo fenomeno sia oltremodo accentuato in Italia, ossia in un contesto organizzativo segnato dallo smantellamento della carriera (Tarello 1988; Taruffo 1991), anche se è divenuto ormai visibile anche in Francia (Garapon 1996). In ogni caso, il rafforzamento delle garanzie di indipendenza interna, in seguito all'istituzione di organi di autogoverno della magistratura, e le modificazioni in atto nell'assetto burocratico sembrano ovunque allentare l'influenza dell'élite giudiziaria, ciò che non può che favorire l'emergere ai vari livelli di orientamenti decisionali improntati ad una maggiore autonomia.

3 D'altra parte, il diritto del cittadino di invocare la più alta giurisdizione del paese, talvolta esplicitamente sancito dalle stesse carte costituzionali, e il carico di lavoro che ciò comporta spiegano la composizione pletorica delle corti supreme (Shapiro 1980). Il fatto che siano formate da centinaia di giudici, suddivisi in sezioni diverse, appare spesso paradossale agli occhi degli osservatori anglosassoni, tenuto conto che il loro compito precipuo dovrebbe appunto consistere nell'assicurare un'interpretazione delle norme uniforme e coerente (Merryman 1985), un compito questo che sembrano infatti svolgere con difficoltà crescenti. 


\section{L'accesso alla giustizia: le condizioni generali}

La descrizione degli assetti giurisdizionali offre dunque un quadro sufficientemente preciso della competenza del sistema giudiziario nei diversi paesi, ossia dei casi che il giudice può essere chiamato a decidere. Come abbiamo appena visto, il raggio d'intervento del giudice costituisce un fattore di cui si deve tener conto per valutare l'incidenza relativa del giudiziario rispetto alle altre istituzioni politiche. Tuttavia, per comprendere il ruolo che questi effettivamente assume, è necessario considerare anche come e in quale misura questa ampiezza potenziale può realizzarsi. Si tratta in altre parole di spostare l'attenzione su un fascio di elementi relativi al modo in cui il giudiziario può essere in concreto attivato e che vengono solitamente riassunti nella formula «accesso alla giustizia». Il nostro prossimo passo consiste quindi nel passare in rassegna i principali meccanismi, formali e non, che possono incoraggiare o meno il ricorso al giudice e che dunque concorrono a modellare il flusso delle domande rivolte al sistema dai singoli e, eventualmente, dai gruppi. In seguito prenderemo in esame il ruolo giocato da alcuni attori che, in forza della loro collocazione istituzionale, sono in grado di controllare le vie d'accesso al sistema e di filtrare, se non addirittura di determinare, $\mathrm{i}$ casi che questo dovrà trattare.

Diversamente da altri attori istituzionali, il giudice è sempre tenuto a prendere una decisione sui casi che gli vengono assegnati: il giudice non può «denegare giustizia». Laddove l'attenzione degli apparati legislativi o amministrativi può essere più facilmente sollecitata da soggetti in grado di controllare determinate risorse strategiche - come ad esempio la disponibilità di canali privilegiati di comunicazione - ogni cittadino ha la possibilità, quando non il diritto formalmente sancito (Cappelletti 1989, 222), di adire un giudice con l'aspettativa legittima di ottenerne una risposta. Questo principio generale fa del giudiziario un' istituzione naturalmente «aperta», o quanto meno più accessibile di altre istituzioni politiche. Diversamente da queste, il giudiziario si è in effetti sviluppato per processare domande individuali e, almeno in linea di principio, i singoli non hanno bisogno del sostegno di un gruppo o di un movimento per invocare l'intervento di un giudice (Peltason 1968). È noto del resto che un'azione legale, anche se intentata su base individuale, può sollevare questioni più generali e che toccano in qualche misura gli interessi della collettività. Il punto da mettere in evidenza è 
che ciascuno dispone in astratto della possibilità di azionare il sistema, di utilizzare il processo per far valere i propri diritti o anche per articolare domande politiche, e di ottenere comunque una decisione che si imporrà almeno sulle parti rappresentate in giudizio, ma che non di rado avrà ricadute di ben più ampia portata. Benché si riconosca che le opportunità di accesso sono distribuite in modo ineguale, il ricorso al giudice è infatti considerato un importante strumento di partecipazione politica (Zemans 1983; Waltman 1988, 218-219), che si presta a legittimare, almeno in certa misura, il ruolo - esso stesso politico - che la giustizia sta assumendo. In realtà, tutti i sistemi pongono dei limiti all'accesso, che possono assumere forme diverse e rispondere ad obiettivi altrettanto diversi ${ }^{4}$. E per questa ragione che i meccanismi che concorrono a definire le dimensioni effettive del ricorso al giudice costituiscono altrettante variabili di importanza critica per stimarne l'incidenza politica relativa (Sarat e Grossman 1975).

Un primo insieme di elementi attiene alla trasformazione di una conflitto in un'azione legale. Si tratta cioè del primo segmento di un processo molto articolato che ha inizio quando un conflitto viene tradotto in termini giuridici e che, passando di solito attraverso la consulenza di un avvocato, può poi approdare alla formulazione di una domanda innanzi al giudice competente (Blankenburg 1994, 691; Friedman 1994). In generale la «mobilitazione» delle strutture giurisdizionali dipende da molti fattori: gli atteggiamenti e i valori condivisi relativamente al rapporto fra diritto e cittadino, ossia il tipo di cultura giuridica; le alternative a disposizione delle controparti in lite; $i$ cost $i$ che queste comportano; le aspettative riguardanti le risposte che le istituzioni specializzate nella gestione dei conflitti, e dunque non solo il giudiziario, possono fornire; e soprattutto le risorse disponibili per mobilitare effettivamente tali istituzioni (Grossman e Sarat 1981, 77). Quest'ultima osservazione ci porta a sottolineare l'importanza della diffusione di conoscenze sulle istituzioni, le procedure e le forme di tutela che i singoli possono invocare in difesa dei propri diritti. Il grado di «alfabeti-

4 Dall'esigenza di ridurre il volume complessivo del contenzioso per non sovraccaricare il sistema, attraverso l'istituzione di meccanismi alternativi di soluzione delle dispute, sino a quella di consentire la rappresentanza in giudizio ai soli attori che possano mostrare un interesse diretto e personale alla decisione, escludendo pertanto soggetti individuali e collettivi che potrebbero esserne egualmente toccati (Marshall 1976). 
smo giuridico» costituisce infatti una sorta di precondizione necessaria per accedere alla giustizia. Ed è evidente che, per quanto legato ai livelli di istruzione (Waltman 1988), esso dipende anche dagli investimenti pubblici per aprire il sistema ed informare i potenziali litiganti sui loro diritti così come sugli strumenti per farli valere. La decisione di intentare un'azione legale deve però essere messa in relazione anche con altri fattori, che potremmo egualmente classificare sotto la voce «offerta di giustizia», dal momento che riguardano il rendimento della macchina giudiziaria in rapporto alla domanda che essa riceve, e che sembrano costituire un elemento di variazione ben più importante di quelli legati al tipo di cultura giuridica. Tra tali fattori il primo è certamente costituito dai costi, da intendersi peraltro in senso lato. I costi in questione non includono solamente le spese processuali o di rappresentenza legale ma anche quelli sociali e persino psicologici. Per quanto importanti, questi ultimi giocano un ruolo che può però essere più facilmente apprezzato in relazione ai casi specifici, ossia al tipo di controversia e alla natura dei rapporti esistenti fra le controparti ${ }^{5}$, e si situano dunque al di fuori della nostra analisi. Quanto ai costi economici del processo, seguendo la cronologia indicata $\mathrm{da}$ Cappelletti (1981a, 7-11), essi hanno costituito il primo ostacolo con cui il movimento per l'accesso alla giustizia si è dovuto misurare su scala internazionale. Basti pensare che l'approccio tradizionale, legato alle politiche di laissez faire, si limitava a dispensare gli indigenti dagli oneri processuali, imponendo agli avvocati l'obbligo di provvedere all'assistenza legale su base prettamente volontaria. Nella maggior parte dei paesi lo sviluppo dello stato sociale ha visto l'adozione di una serie di riforme che hanno gradualmente sostituito tale approccio, col riconoscere agli indigenti il diritto di essere assistiti prima e durante l'eventuale processo, attraverso la creazione di servizi di consulenza giuridica e di assistenza giudiziaria.

Come si è detto, la frequenza e la diffusione del ricorso al giudice sono da mettere in relazione anche con l'esistenza e il grado di accessibilità di altre istituzioni specializzate nella gestione dei conflitti, o che comunque adempiano ad una funzio-

5 Come osserva Crawford, «un'azione legale è più probabile nel caso in cui le due controparti non intrattengano alcuna relazione personale e laddove il mantenimento di buoni rapporti appare secondario» $(1994,2)$. Al riguardo Blankenburg utilizza i concetti di «distanza sociale» e di «formalizzazione del rapporto» $(1994,693)$. 
ne di controllo sociale (Eckhoff 1976; Shapiro 1981). Nella misura in cui queste riescono a rappresentare una efficace alternativa funzionale alla giurisdizione, possono entrare in concorrenza col sistema giudiziario ed eroderne almeno in parte il monopolio della soluzione delle dispute. Questo è quanto si verifica per alcuni tipi di controversie, la cui diffusione è generalmente associata al grado di sviluppo economico, come ad esempio le transazioni commerciali a livello nazionale e transnazionale. Si tratta di dispute che con una certa frequenza tendono a sfuggire al sistema giudiziario per rivolgersi a forme di composizione che, come l'arbitrato, non compromettono le relazioni future tra litiganti e assicurano nel contempo una decisione non soltanto più rapida ma anche confidenziale, benché sia generalmente necessario comparire davanti ad un giudice per ottenere un titolo esecutivo. Non meno importante, quanto ai possibili effetti sul raggio dell'intervento giudiziario, è il ruolo giocato da un numero crescente di organismi non giurisdizionali e che tuttavia tendono a riprodurre le procedure di decisione, e dunque le garanzie, che sono proprie alla giurisdizione. È il caso delle cosiddette autorità amministrative indipendenti, istituite in un numero crescente di paesi. Come la denominazione sta ad indicare, la loro collocazione istituzionale presenta alcune analogie con quella del giudice e simili sono anche gli obiettivi perseguiti nell'istituirle. Tali autorità sono infatti concepite come agenti imparziali di decisione, la cui legittimazione si fonda essenzialmente sulla competenza tecnica ${ }^{6}$. Istituite con l'intento di sottrarre le relative decisioni alle contingenze della politica e alle pressioni partigiane in settori ritenuti nevralgici, come ad esempio le comunicazioni e la concorrenza, la loro diffusione può effettivamente ridurre il raggio d'intervento potenziale del giudi$\mathrm{ce}^{7}$, con conseguenze in certa misura simili a quelle osservate a proposito della frammentazione giurisdizionale, anche se le relative decisioni sono generalmente suscettibili di ricorso davanti al giudice. Da ultimo, anche i processi di concertazione neocorporativa possono agire come surrogati funzionali del giudi-

6 I parallelismi tra giurisdizione e autorità amministrative indipendenti sono analizzati da Majone (1994). In termini più generali, questo fenomeno deve essere messo in relazione con un fenomeno di più ampia portata, ossia la tendenza a replicare alcuni tratti tipici del procedimento giudiziario in settori estranei alla giurisdizione (Vallinder 1995).

7 Questa almeno è l'opinione espressa da Haenel e Harthuis (1991, 44-45). 
ziario nel trattare conflitti socialmente rilevanti e ridurne così l'incidenza politica relativa. I meccanismi istituzionali che garantiscono ai gruppi di volta in volta interessati l'opportunità, quando non il diritto formalmente sancito, di partecipare alla formulazione delle politiche pubbliche operano infatti come camere di compensazione, e di mediazione, fra gli interessi in gioco prima che le decisioni siano adottate. I gruppi diventano in tal modo interlocutori stabili delle istituzioni di governo e particolarmente della burocrazia durante tutto il processo di formulazione e messa in opera delle politiche. Fondandosi sul principio della «condivisione del potere», gli assetti neo-corporativi si prestano dunque ad assorbire preventivamente una parte consistente dei conflitti potenziali, di modo che, in linea di principio, le azioni legali nei settori di policy caratterizzati da questo tipo di gestione vengono intentate solo in caso di fallimento di tali procedure consensuali.

Più in generale, la capacità di risposta delle altre componenti del sistema politico è uno degli elementi utili per valutare l'incidenza del giudiziario (Guarnieri e Pederzoli 1996, cap. 4). La propensione a mobilitare le strutture giurisdizionali dipende infatti anche dalla loro utilità relativa, così come percepita dai singoli e dai gruppi (Becker 1970, 347; Friedman 1976). In effetti, a fianco di quella che si potrebbe definire come «fuga dalla giurisdizione», alimentata dagli elevati costi sociali ed economici del processo, dal ritardo con cui spesso le risposte vengono fornite o dalle strategie poste in essere dal sistema politico per ridurre l'area di intervento potenziale del giudice, è possibile osservare anche fenomeni che muovono nella direzione opposta. Si assiste cioè alla tendenza a trasferire nell'arena giudiziaria domande, che non hanno trovato ascolto in altre sedi, dirette a far valere diritti o anche ad affermare nuove pretese, la cui portata va oltre l'interesse personale del singolo attore che promuove l'azione. Simili controversie, che nei sistemi anglosassoni sono indicate come public interest litigation (Chayes 1976), possono assumere forme molto diverse. Può trattarsi di cause intentate su base individuale attraverso le normali procedure previste per il contenzioso penale, civile e amministrativo, ma aventi ad oggetto la tutela di interessi diffusi, o ancora di cause iniziate da soggetti collettivi ricorrendo a nuove strategie processuali. La tendenza ad utilizzare l'azione legale - tradizionalmente concepita per dirimere dispute private - come mezzo per articolare domande politiche o per modificare politiche pubbli- 
che è un fenomeno tutt'altro che recente, come documentano del resto le ormai classiche osservazioni di Tocqueville sulla democrazia americana. E vero però che tale fenomeno ha conosciuto una notevole accelerazione nelle democrazie contemporanee, grazie soprattutto alla crescente sottoposizione degli atti pubblici all'esame di una autorità giudiziaria indipendente, cui i singoli possono rivolgere le loro contestazioni. Comunque sia, la propensione ad utilizzare il processo in questo senso, essa stessa un indizio della politicità della funzione giudiziaria, non dipende solo dalle risposte fornite dalle altre istituzioni. Per la parte che qui interessa, essa è chiaramente influenzata anche dalle modalità di funzionamento delle istituzioni giudiziarie, dal loro rendimento, dai rimedi che il giudice può ordinare e dalle norme che disciplinano l'accesso, particolarmente quelle in tema di diritto d'azione. In altre parole, si tratta ora di considerare quello che è stato definito come l'ostacolo propriamente processuale al movimento di accesso alla giustizia, e cioè lo scarto riscontrabile tra le forme tradizionali di legittimazione ad agire, che generalmente consentono l'azione a soggetti individuali aventi un interesse diretto e personale, e il tentativo di perseguire nuovi obiettivi di tutela collettiva (Cappelletti 1994, 71-102).

In generale, le soglie d'accesso al sistema giudiziario cominciano ad abbassarsi negli Stati Uniti a partire dagli anni '60. Concorrono in questo senso sia l'attivismo mostrato dall'avvocatura sia, soprattutto, gli orientamenti favorevoli assunti dalla giurisprudenza - specie dalla Corte suprema federale sotto la presidenza Warren - nei confronti delle class actions, particolarmente in materia di diritti civili (Grossman e Sarat 1981, 82). I risultati ottenuti in tale periodo dal movimento per la difesa delle persone di colore, attraverso forme di rappresentanza collettiva in giudizio a tutela di interessi altrimenti troppo disorganizzati e deboli, hanno fornito un modello che è stato in seguito replicato con successo in altri settori. In Europa, gli sviluppi in questa direzione sono più lenti e tardivi e cominciano a manifestarsi all'inizio degli anni '70, soprattutto in materie come la difesa dell'ambiente e la tutela dei consumatori. Fra le eccezioni di rilievo figura però l'Inghilterra, in cui la titolarità dell'azione resta, in linea di principio, limitata ai soli soggetti che possono comprovare un interesse diretto e personale alla controversia (Feldman 1992). Le formule adottate per assicurare la protezione di interessi collettivi e diffusi prendono così atto implicita- 
mente delle carenze denotate dalla figura del pubblico ministero. Benché istituzionalmente chiamato a rappresentare l'interesse generale anche in molti procedimenti civili, il pubblico ministero ha generalmente mostrato scarsa propensione ad assumere questa responsabilità, limitando il proprio raggio d'azione al ben più familiare terreno penale (Cappelletti 1989, 275-278). Al di là delle inevitabili differenze ravvisabili nei singoli ordinamenti processuali, sarà sufficiente ricordare che le soluzioni adottate consistono per lo più nel riconoscere ad associazioni private il diritto di agire in giudizio in favore di soggetti non identificabili individualmente, attraverso forme di rappresentanza pressoché sconosciute alla tradizione continentale, come ad esempio le actions en interet collectif in Francia o le Verbandsklagen in Germania.

Benché importanti, le opportunità d'accesso alla giustizia, così come definite dal quadro normativo, non costituiscono certamente la sola condizione da soddisfare affinché il processo sia effettivamente utilizzato da singoli e gruppi come strumento per formulare domande politiche. Come già accennato, non meno importante è che $\mathrm{i}$ gruppi percepiscano il giudiziario come uno dei possibili canali di articolazione delle domande politiche. Del pari è evidente che anche le reazioni delle corti a tali domande - particolarmente quelle promosse nell'intento esplicito di sondarne gli umori, i cosiddetti «processi pilota»hanno un peso in questo senso. Gli orientamenti decisionali del giudice possono qui diventare determinanti. $\grave{E}$ chiaro che un giudice interventista, che si comporta come attore politico autonomo e che viene percepito come tale, può giocare un ruolo certamente più incisivo di quello del suo antagonista idealtipico, il giudice «bocca della legge» (Guarnieri e Pederzoli 1996 , cap. 2). Non va inoltre dimenticato che l'eventuale risposta favorevole fornita dal giudiziario può costituire un segnale di disponibilità a trattare certi problemi: le corti possono dunque incoraggiare questo uso del processo ed influire, almeno in certa misura, sulle domande che vengono loro rivolte, concorrendo così a definire la propria agenda. Inoltre, man mano che il giudice diviene un referente per i gruppi d'interesse, è anche probabile che le decisioni rese nelle materie in cui più frequente è il suo intervento tendano a perdere il carattere discontinuo che altrimenti le caratterizza - essendo passivo, il giudice non può scegliere i casi su cui pronunciarsi - dando luogo a politiche giurisprudenziali relativamente coerenti e dunque teorica- 
mente capaci di avere un impatto maggiore (Weiler 1968, 446).

L'importanza da attribuire alla capacità di risposta del sistema giudiziario e agli orientamenti di ruolo della magistratura non deve comunque indurre a sottovalutare il peso esercitato da un altro segmento delle professioni legali, gli avvocati. In qualità di tramite pressoché obbligato fra cittadino e giustizia, l'avvocatura assume un ruolo per certi aspetti simile a quello del pubblico ministero, ossia di «guardiano» delle vie d'accesso alla giustizia, particolarmente nel settore civile. Anche gli orientamenti dell'avvocatura concorrono in effetti a modellare il flusso di domande che pervengono alle corti sotto un duplice profilo: quantitativo, dal momento che possono ad esempio incentivare la mediazione piuttosto che lo scontro frontale in giudizio, e qualitativo, poiché la definizione degli obiettivi e l'impostazione delle strategie processuali da seguire sono per lo più lasciati alla valutazione professionale dell'avvocato. Un aspetto che merita di essere sottolineato è costituito dalla disponibilità dell'avvocatura ad impegnarsi nella political advocacy, ossia un'attività di consulenza e, se necessario, di patrocinio legale gratuiti e condotta in favore di soggetti socialmente deboli e privi di risorse organizzative. Il profilo socio-economico della professione e la sua stessa struttura, ad esempio la diffusione di studi legali di grandi dimensioni o viceversa la frammentazione in unità di piccole dimensioni, sono alcuni tra gli elementi suscettibili di influire sulla propensione ad intraprendere attività di questo tipo (Blankenburg e Cooper 1982, 264-265). Va però ricordato che anche le norme che disciplinano il versante economico del rapporto tra professionista e cliente sono importanti ${ }^{8}$.

Da ultimo non va trascurato che anche la struttura del procedimento giudiziario e i margini di intervento di cui il giudice dispone sono da annoverarsi fra $\mathrm{i}$ fattori che possono in una certa misura influire sulla decisione di avviare o meno un'azione legale. Come vedremo meglio fra poco, si tratta di elementi che incidono sulla capacità dei litiganti di governare il processo che li vede coinvolti e, dunque, il suo esito. Prima di prendere in esame questo punto, dobbiamo però considerare il ruolo gioca-

8 Basti pensare alla diffusione negli Stati Uniti, e in misura inferiore in Europa, dei cosiddetti «patti quota-lite», in base ai quali l'avvocato percepisce un onorario, calcolato in percentuale sulle somme ottenute a titolo di risarcimento, solo in caso di esito favorevole della causa (Hazard e Taruffo 1993, 114-115). 
to $\mathrm{da}$ un attore istituzionalmente chiamato a presidiare le vie d'accesso al sistema giudiziario, quanto meno nel settore penale, il pubblico ministero.

\section{Il pubblico ministero}

L'accesso al sistema giudiziario, soprattutto penale, è fortemente influenzato dal pubblico ministero. Nei regimi democratici è di solito presente un attore incaricato istituzionalmente di richiedere al giudice l'applicazione della legge e che, quindi, partecipa al processo di applicazione delle norme giuridiche e, in generale, alla funzione politica che definisce lo stato: la pretesa di esercitare su un certo territorio il monopolio della violenza legittima. Anche in questo campo la tradizione di civil law si differenza da quella di common law. L'origine del pubblico ministero può essere fatta risalire alla Francia del XIV secolo (Perrot 1993, 276), ma è dopo la riorganizzazione effettuata da Napoleone all'inizio del XIX secolo che l'istituto - cioè un corpo di funzionari o magistrati incaricati di esercitare l'azione penale e di difendere l'interesse pubblico - si diffonde negli altri paesi dell'Europa continentale. Il pubblico ministero viene così collocato all'interno di una struttura processuale in cui l'obiettivo principale dell'azione penale è quello di contribuire a realizzare la politica dello stato, indipendentemente dall'atteggiamento della vittima e dell'accusato (Damaska 1991, 314). E qui dove la tradizione burocratica è più forte - che l'assimilazione fra giudice e pubblico ministero è più elevata e che le modalità di esercizio dell'azione penale tendono ad essere maggiormente ispirate al principio di imparzialità: il pubblico ministero viene spesso definito una «parte imparziale» che svolge le funzioni d'accusa nell'interesse esclusivo dello stato, fatto che lo pone al di sopra delle parti private (Guarnieri 1984). Nei paesi di common law, come vedremo, dove la funzione del processo penale è sempre stata piuttosto quella di risolvere controversie, la situazione è stata tradizionalmente differente: il pubblico ministero tende qui ad essere considerato il rappresentante della vittima (Damaska 1991, 183). Addirittura, per lungo tempo, in Inghilterra esercitare l'azione penale è stato compito soprattutto della polizia o di privati cittadini.

La crescita dell'intervento statale sulla società ha avuto effetti anche sul ruolo del pubblico ministero nel processo pena- 
le, dal momento che i settori della vita sociale regolati dallo stato sono notevolmente aumentati e così anche le probabilità che si verifichi una violazione della legge. Nel frattempo, anche il modo con cui vengono definiti i reati è mutato: «i testi che stabiliscono una violazione cessano di avere la precisione che un tempo garantiva un'interpretazione rigida della legge. Le infrazioni vengono definite in modo sempre più ampio, e tocca al giudice qualificare $\mathrm{i}$ fatti all'interno del quadro fluttuante che gli viene offerto da testi imprecisi »(Maillard 1994, 106). D'altra parte, la crescita del ruolo del pubblico ministero innescata da questi sviluppi rende di maggior rilievo il tema della sua autonomia, dato che, dove presente, il suo tradizionale rapporto con l'esecutivo può diventare un problema. Infatti, tale dipendenza può far sospettare che in certi settori - cioè dove le azioni dell'esecutivo sono in gioco - la legge non venga applicata con sufficiente energia. E un atteggiamento che dipende anche dal grado di fiducia dell'opinione pubblica nei confronti dell'esecutivo, ma che in ogni caso spinge a garantire una certa autonomia a chi esercita l'azione penale.

Il pubblico ministero condiziona l'incidenza politica del sistema giudiziario influendo sui casi che vi hanno accesso. I compiti che i sistemi giudiziari contemporanei gli affidano sono numerosi e variano da un sistema all'altro, ma sono importanti soprattutto quelli che definiscono il suo ruolo nella giustizia penale: per esempio, dare inizio alla procedura, controllare lo svolgimento delle inchieste, decidere se esercitare l'azione penale o proporre l'archiviazione, in certi casi seguire lo sviluppo dell'istruttoria diretta da un giudice, rappresentare l'accusa in udienza, ecc. Il sistema giudiziario penale non può dunque non dipendere dal pubblico ministero e dalla sua discrezionalità: infatti non potrebbe funzionare senza qualche filtro, e quindi senza qualche forma di discrezionalità. Tutti i sistemi giudiziari penali devono tener conto, apertamente o meno, di questa esigenza. Gli assetti del pubblico ministero dei vari paesi si distinguono fra loro a seconda dei limiti e dei controlli imposti alla sua discrezionalità (Weigend 1983b, 1296; Díez-Picazo 1995). In questo campo è la struttura che conta, piuttosto che i grandi principi della procedura. Per esempio, non bisogna sopravvalutare l'importanza del monopolio pubblico dell'azione penale. Anche in un sistema in cui l'azione privata è ammessa, il pubblico ministero dispone di risorse molto più ampie di qualunque cittadino e gode pertanto di un quasi-monopolio di fatto 
dell'azione penale. La stessa distinzione fra obbligatorietà e opportunità nell'esercizio dell'azione penale - in altre parole, il riconoscimento o meno nel sistema processuale di un potere discrezionale in capo al pubblico ministero - non deve essere sopravvalutata. Anche se non va trascurato il valore simbolico di questi principi, i comportamenti concreti sembrano restituirci in realtà un'immagine abbastanza differente da quella formalmente prevista. La discrezionalità del pubblico ministero non dipende solo dai principi della procedura ma anche - anzi, soprattutto - dal suo assetto organizzativo, cioè dai compiti che è chiamato a svolgere nel sistema giudiziario, dai controlli sul suo operato e dai caratteri del personale che vi lavora (Weigend 1983b; Guarnieri 1984, 145 ss.; Damaska 1991; Di Federico 1995). Quindi, compiti numerosi e maldefiniti, controlli inefficaci e un personale culturalmente poco omogeneo, ma dotato di forti garanzie, renderanno probabile la presenza di forti variazioni nel tipo di azioni penali effettivamente esercitate.

In realtà, per valutare il ruolo svolto nel sistema giudiziario dal pubblico ministero bisogna innanzitutto considerarne i rapporti con gli attori principali e in primo luogo col giudice. Per esempio, il fatto che pubblico ministero e giudice appartengano allo stesso corpo ha senz'altro delle conseguenze, anche se non univoche. In linea di principio, una magistratura che svolge le funzioni sia giudicanti sia requirenti dispone di maggiore potere: la posizione del pubblico ministero ne è rafforzata, dato che, al contrario della difesa, è legato organizzativamente - e anche culturalmente - al giudice (Weigend 1983b, 1297). D'altra parte, molto dipende anche dalle garanzie. Nelle magistrature napoleoniche un pubblico ministero subordinato rigidamente al governo è la strada attraverso la quale l'esecutivo esercita influenza su tutta la magistratura. Le relazioni con il giudice sono poi influenzate anche dalla struttura del processo. Ci riferiamo al processo penale, perché è qui che di solito il ruolo del pubblico ministero è più importante. Come vedremo, dove presente, è soprattutto la relazione col giudice istruttore che deve essere tenuta in conto: chi svolge la parte più importante nella fase pre-dibattimentale? La risposta dipende dalle norme giuridiche ma soprattutto dalla prassi (Weigend 1983a). Infine, un altro elemento è il rapporto con la polizia. La polizia, e specie la polizia giudiziaria, svolge un ruolo molto importante nel sistema giudiziario penale, dal momento che il suo compito principale è indagare sui reati. La capacità del pubblico ministero 
di giocare un ruolo attivo e, quindi, l'influenza che è in grado di esercitare sulla giustizia penale dipendono molto dalla sua capacità di orientare i comportamenti della polizia (Weigend 1983 b) $)^{9}$.

Ad ogni modo, lo status del pubblico ministero è probabilmente l'elemento più importante. Di norma, gli assetti prevalenti nei regimi democratici tendono a presentare forme sia di autonomia sia di responsabilità: autonomia perché le decisioni del pubblico ministero nei casi specifici non devono essere né apparire influenzate da una parte politica, responsabilità a ragione della loro importanza nel processo di attuazione delle politiche penali. Queste due esigenze vengono combinate in modo diverso, ma è evidente che la capacità del pubblico ministero di agire in modo autonomo, così come gli interessi che tenderà a considerare, dipendono molto dai caratteri dell'assetto. Naturalmente, non basta tener conto solo delle garanzie istituzionali. Anche il personale è importante. Ad esempio, è probabile che indipendentemente dalle garanzie - un personale di carriera, che tende a passare tutta la sua vita lavorativa all'interno dell'organizzazione del pubblico ministero, mostrerà un'autonomia minore di un personale che tende a restare all'interno di tale organizzazione per un periodo di tempo limitato, dal momento che può con facilità passare ad altre occupazioni, anche nel settore privato. Infine, anche la struttura è un elemento importante. Un pubblico ministero decentrato e differenziato - come nel caso degli Stati Uniti - con forme diverse di responsabilità, tenderà a favorire l'immissione nel sistema giudiziario di domande abbastanza varie. D'altra parte, la centralizzazione aumenta il potere dell'ufficio ma, rendendolo maggiormente evidente, può facilitare un controllo esterno. In ogni caso, l'articolazione organizzativa è un elemento che influenza il grado e le modalità della responsabilità del pubblico ministero così come la sua disponibilità a rispondere alle domande dell'ambiente.

In generale, l'incidenza politica del sistema giudiziario sarà tanto più elevata quanto più il pubblico ministero sarà autono-

9 Vi sono modelli differenti di relazioni fra polizia e pubblico ministero che vanno dalla presenza di corpi autonomi - la situazione che tende a prevalere nei paesi anglosassoni - alla subordinazione funzionale che caratterizza i paesi di civil law (Feeley e Lazarson 1983; Weigend 1983a). Ma anche in questi paesi la parte svolta dalla polizia nella fase investigativa sembra essere notevole (Goldstein e Marcus 1977; Leigh e Zedner 1992). 
mo dalle altre istituzioni politiche. In tutti i casi considerati con l'eccezione dell'Italia e, in parte, del Portogallo - sono presenti forme di responsabilità politica del pubblico ministero (vedi la tab. 1). Esiste naturalmente una differenza fra un pubblico ministero organizzato in modo unitario e gerarchico e sottoposto al ministro della Giustizia - come, almeno in linea di principio, in Francia - e il caso americano dove una molteplicità di uffici - più o meno autonomi fra loro - sono sottoposti a differenti sollecitazioni da parte dell'ambiente politico. In quest'ultimo caso, infatti, dal momento che non vi è un controllo centralizzato sull'immissione dei casi nel sistema giudiziario penale, la sua incidenza politica sarà senz'altro più elevata che in Francia, mentre in posizione intermedia vanno collocate Inghilterra, Spagna e Germania. L'incidenza sarà poi ancora più forte nel caso del Portogallo, che attribuisce al pubblico ministero ampie garanzie di indipendenza, e soprattutto dell'Italia, dove i magistrati requirenti formano un solo corpo con quelli giudicanti e ne condividono le garanzie.

Come abbiamo accennato, l'importanza del principio di obbligatorietà - o di quello di opportunità - non va invece sopravvalutata. Il fatto che nella maggior parte dei regimi democratici l'azione penale sia guidata dal principio di opportunità non significa che il pubblico ministero possa agire a suo arbitrio, dal momento che vi sono sempre dei controlli sul modo con cui adopera la sua discrezionalità. Paradossalmente, è il principio di obbligatorietà che, in assenza di controlli efficaci, può favorire lo sviluppo di una discrezionalità senza responsabilità, rafforzando così il potere del pubblico ministero e, probabilmente, l'incidenza politica della giustizia. In ogni caso, il ruolo del pubblico ministero deve essere valutato analizzando la sua posizione all'interno del sistema giudiziario e tenendo conto soprattutto dei suoi rapporti con la polizia, da un lato, e il giudice, dall'altro. In altre parole, bisogna considerare anche la struttura del processo.

\section{La struttura del processo}

L'analisi delle forme processuali consente di osservare gli apparati giudiziari in azione, dalla formulazione della domanda di giustizia sino alla decisione che pone fine alla disputa. $\grave{E}$ così possibile ricostruire nelle sue linee generali il reticolo delle inte- 


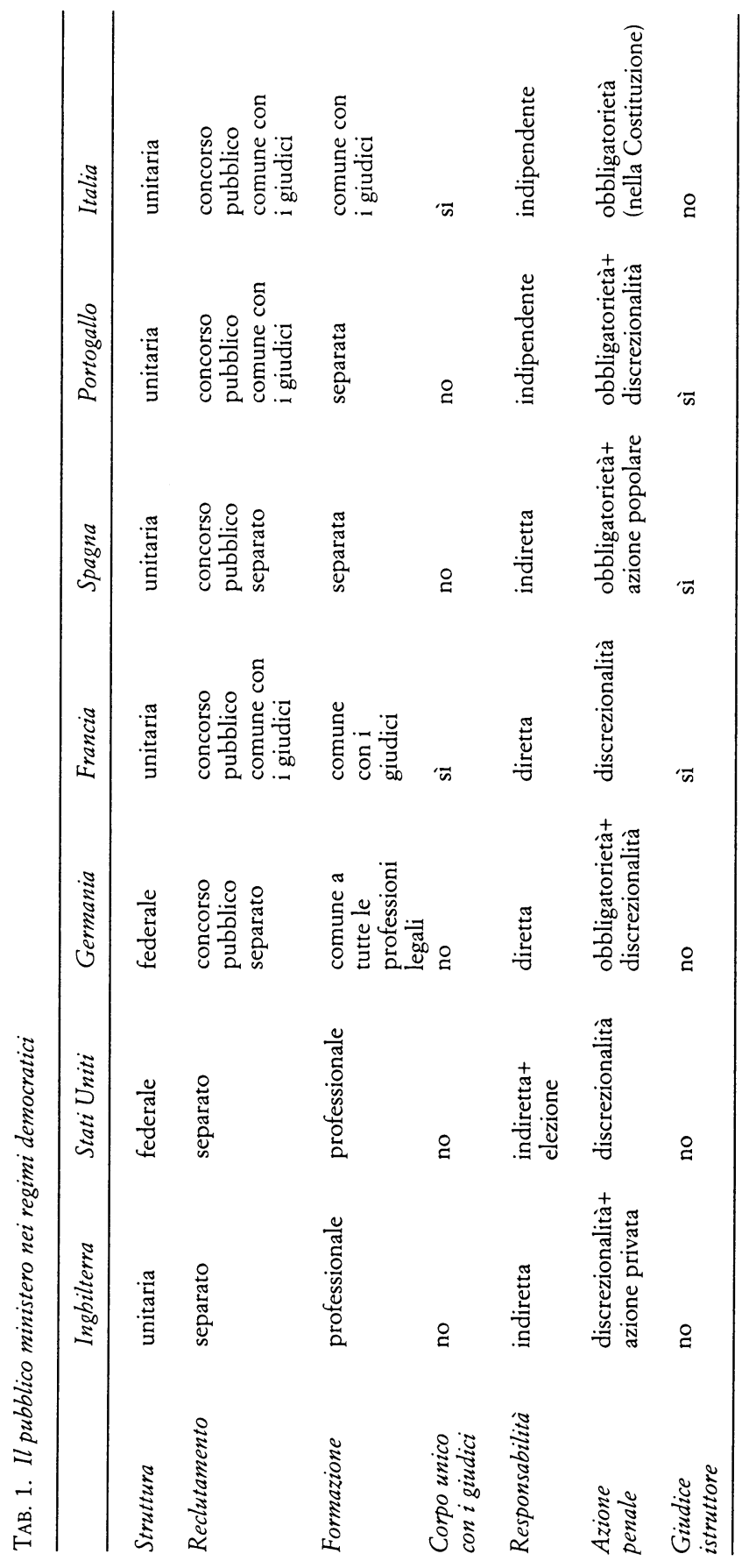


razioni che si stabiliscono fra una pluralità di attori: il giudice, le controparti, e fra queste all'occorrenza il pubblico ministero, le forze di polizia, i testimoni, gli esperti. Va da sé che le strutture realizzate per organizzare e gestire questo insieme di rapporti sono alquanto complesse. Ciò nonostante, nel susseguirsi delle attività processuali che culminano nella sentenza è generalmente possibile isolare due blocchi principali: una fase preliminare, destinata ad istruire o preparare la causa, e il processo propriamente detto, ossia il dibattimento celebrato alla presenza di un giudice, monocratico o collegiale, talvolta affiancato da una giuria laica.

Per quanto importante, il grado di articolazione della procedura non è tuttavia né il primo né il più rilevante criterio da seguire per orientarsi nella complessità delle formule processuali osservabili nelle democrazie contemporanee. Poiché siamo interessati in primo luogo a comprendere la parte assegnata al giudice, soprattutto la gamma e il peso specifico delle funzioni che egli può svolgere, è invece più utile soffermarsi sulla ripartizione delle competenze o, per meglio dire, dei poteri tra i diversi attori processuali. Le norme che definiscono la struttura e le modalità di svolgimento del processo non costituiscono solo uno strumento per razionalizzare l'esercizio del potere, così da legittimare la sentenza (Calamandrei 1965, 628). Tali norme provvedono anche ad allocare potere, ossia a ripartire le risorse che consentono di controllare lo svolgimento dell'istanza fra i soggetti che vi partecipano (Peltason 1968). Nell'ambito dei vincoli posti dalla legge esistono infatti margini d'azione piuttosto ampi, esplicitamente lasciati all'apprezzamento dei singoli attori - giudice e controparti - e che riguardano non soltanto le questioni sostanziali da dibattere in giudizio ma anche il tipo di procedura da seguire. Ad esempio, in diversi paesi l'imputato si vede riconoscere la possibilità di optare per una procedura ordinaria oppure per un rito abbreviato, o ancora di essere giudicato o meno da una giuria. Considerato da questa angolazione, e come già osservava Calamandrei $(1965,638)$, il processo altro non è se non una sorta di canovaccio, i cui contenuti sono definiti dagli attori che vi partecipano. È allora importante chiarire in primo luogo quali sono i soggetti di volta in volta legittimati a prendere le decisioni rilevanti e che dunque sono in grado di governare la sequenza degli eventi processuali sotto il duplice profilo sostanziale e formale.

La partizione classica tra processo inquisitorio ed accusato- 
rio, che abbraccia sia la giustizia civile sia quella penale, riflette essenzialmente una diversa allocazione dei poteri (Damaska 1975; Weigend 1983a, 537). Mentre l'uno pone in primo piano la figura del giudice, l'altro esalta invece il ruolo delle controparti e soprattutto dei loro rappresentanti legali, gli avvocati. Seguendo le classificazioni abitualmente impiegate nelle analisi comparate, si potrebbe parlare anche di un processo adversary contrapposto ad uno non-adversary (Fanchiotti 1993). Si tratta di due formule che hanno forse il pregio di meglio inquadrare le differenze riscontrabili quanto alla struttura delle interazioni fra gli attori e agli obiettivi che perseguono. Come si vedrà fra poco, questi ultimi costituiscono del resto un'importante dimensione d'analisi. Benché nella loro versione pura questi due modelli rappresentino solo gli estremi di un continuum, lungo il quale si collocano i casi concretamente osservabili, è ugualmente utile vederne più da vicino $i$ rispettivi tratti tipici.

La forma accusatoria o adversary, che storicamente sembra essersi affermata per prima, sostituendo le più antiche forme della faida o del giudizio divino, e che conserva in effetti alcune tracce di tale origine (Stein 1987), si basa sul confronto dialettico tra $i$ due litiganti $o$, più precisamente, tra i loro rappresentanti legali. Essendo sostanzialmente concepito come «affare delle parti», che sole possono decidere delle strategie migliori per argomentare e sostenere le rispettive pretese, questo tipo di processo concentra la maggior parte delle risorse nelle mani degli avvocati, che ne diventano così gli autentici protagonisti. Spetta ad essi istruire la causa, definire le questioni di fatto e di diritto da sottoporre al giudice, raccogliere i mezzi di prova e persino condurre l'escussione dei testimoni prima e durante il dibattimento. Poiché l'allocazione dei poteri processuali tende a configurare lo schema di un gioco a somma zero, l'ampiezza dei poteri d'iniziativa, di impulso e di direzione del procedimento in tal modo devoluti alle parti comporta una pressoché parallela contrazione delle prerogative del giudice. Questi si vede infatti attribuire il ruolo di arbitro neutrale e sostanzialmente passivo, il cui compito consiste nel vigilare sul rispetto delle regole destinate ad assicurare l'equilibrio delle opportunità tra i due antagonisti. Il giudice non può cioè interferire direttamente sul confronto tra le controparti, se non a rischio di alterare tale equilibrio e di vedere seriamente incrinata la propria immagine di terzo distaccato ed imparziale.

Quando si passa a considerare l'assetto inquisitorio o non- 
adversary si assiste al ribaltamento della logica che pone le parti al centro del processo. Una volta avviata l'azione legale, l'istan$\mathrm{za}$, anche nel contenzioso civile, tende a perdere la sua natura privata per divenire non più un affare delle parti, bensì dello stato. In quanto tale, il processo deve essere governato da un giudice-funzionario, chiamato a rappresentare un interesse che trascende quello dei litiganti e che deve all'occorrenza essere fatto valere anche al di là della loro volontà. Spetta dunque al giudice assumere il controllo e la direzione dell'intera sequenza processuale, dall'istruttoria sino al dibattimento. Il ruolo chiaramente attivo che gli viene affidato spiega l'ampiezza dei suoi poteri, particolarmente nella fase preliminare, che dipende tendenzialmente dalla sua sola iniziativa. Ma il giudice dispone anche di importanti prerogative nel corso del dibattimento, prima fra tutte quella di interrogare personalmente le parti e i testimoni. In breve, i due modelli sin qui delineati e che, vale la pena di ricordarlo, altro non sono se non i poli di un continuum, oppongono un processo che lascia alle parti stesse il compito di amministrare la disputa ad un processo che invece delega tale funzione ad un agente pubblico, il giudice.

A questo punto è lecito chiedersi quali sono i fattori che concorrono a spiegare questa diversa allocazione dei poteri fra il giudice e le parti. Come si è detto, la natura specifica degli obiettivi assegnati al processo costituisce un utile punto di partenza. In termini generali il processo può essere considerato uno strumento, se si vuole alquanto sofisticato, di controllo sociale (Shapiro 1981). Il suo obiettivo prioritario e ovunque condiviso resta in effetti quello di dirimere le controversie in modo pacifico. Ma il processo si presta anche ad assumere un'ulteriore funzione, che è del resto strettamente legata alla natura autoritativa della sentenza. Può cioè diventare anche uno strumento per affermare o ribadire valori, principi ed obiettivi di più vasta portata, che all'occorrenza possono travalicare gli interessi particolari dibattuti in giudizio. Seguendo una classificazione proposta di recente dall'analisi comparata, possiamo allora distinguere tra un processo il cui obiettivo è circoscritto alla semplice «soluzione delle dispute» ed un processo le cui finalità si collocano ad un livello più generale, essendo concepito soprattutto come «strumento di messa in opera delle politiche pubbliche» (Damaska 1991).

Dal momento che le forme e gli stili processuali possono essere considerati uno specchio sufficientemente fedele dei valori 
che improntano l'azione pubblica (Cappelletti 1969, 165), questa distinzione può essere collegata al modo di rappresentare il ruolo dello stato nei suoi rapporti con la società, secondo le concezioni di volta in volta dominanti. La linea di confine da tracciarsi in astratto, ma $\mathrm{i}$ casi concreti presentano contorni molto più sfumati, si situa fra uno stato che si limita a definire i propri compiti in modo restrittivo, e la cui sfera d'intervento è dunque alquanto circoscritta, e uno stato che viceversa interpreta la propria missione in modo estensivo ed il cui raggio d'azione può in teoria giungere ad identificarsi con la società stessa. Laddove il primo esalta l'individuo, i suoi diritti e la sua autonomia, il secondo si mostra invece incline a privilegiare la collettività nel suo insieme, il cui interesse generale può imporsi se necessario su quello del singolo. È facile qui riconoscere l'antinomia classica tra un orientamento improntato agli ideali della tradizione liberale, contrapposto ad un orientamento apertamente interventista, che può procedere dalle formule più blande di welfare sino al dirigismo statale proprio dei regimi autoritari. Ritornando al nostro tema, è allora probabile che in uno stato attento ai diritti individuali il processo sia concepito come una sorta di arena istituzionale messa a disposizione dei litiganti, che possono così confrontare in modo largamente autonomo le rispettive pretese. Obiettivo prioritario resta quello di fornire una risposta al problema particolare in tal modo sollevato, attraverso la composizione pacifica della controversia. Sia pure in forma ritualizzata, il processo tende in questo caso a conservare la struttura del duello ad armi pari fra due antagonisti, ciò che giustifica la compressione del ruolo riservato al giudice quale terzo neutrale e passivo. Analogamente, è anche probabile che l'atteggiamento interventista dello stato si riverberi in una certa misura sulle funzioni demandate al processo e sul ruolo, esso stesso interventista, che il giudice è chiamato ad assumere. Poiché in questo caso l'esistenza di una controversia, anche se tra due privati cittadini, tende ad essere considerata come indice di malessere e di disordine sociale, l'istanza non può essere abbandonata al controllo dei litiganti e agli obiettivi particolari e privati che questi si propongono di raggiungere. Il processo perde dunque la fisionomia propria dello scontro di parte per assumere piuttosto il profilo di un'inchiesta pubblica affidata ad un giudice-funzionario, cui si chiede di intervenire in un conflitto che chiama in causa, in modo diretto o indiretto, gli interessi della collettività. L'obiettivo perseguito consiste in tal caso nel 
ristabilire un ordine giuridico violato, facendo valere l'interesse generale anche al di là delle domande specifiche formulate dai litiganti (Damaska 1991).

L'ampiezza dei poteri devoluti al giudice tende dunque ad ampliarsi ovvero a restringersi anche a seconda delle funzioni assegnate al processo, che a loro volta risentono delle diverse ideologie che improntano l'azione dei poteri pubblici in un sistema politico dato. Queste stesse ideologie tendono del pari a riflettersi sulla struttura della giurisdizione e sull'organizzazione giudiziaria. Come abbiamo visto, le strutture propriamente piramidali, che attribuiscono alle corti supreme una funzione di controllo sul lavoro svolto ai livelli inferiori, sono di frequente associate a corpi giudiziari di impianto burocratico, il cui ordine essenzialmente gerarchico costituisce del resto un'efficace cinghia di trasmissione dell'autorità. Di converso le magistrature professionali, nelle quali l'ordine gerarchico è ancora embrionale, sono generalmente associate ad assetti giurisdizionali in cui la dimensione orizzontale prevale su quella verticale e che dunque lasciano maggiore autonomia ai vari livelli di giudizio. $\grave{E}$ bene guardarsi, ad ogni modo, dalla tentazione di stabilire dei rapporti di necessità tra questi elementi, anche perché è possibile osservare contesti che poggiano su «combinazioni» diverse da queste. Le analisi comparate mostrano nondimeno che il processo di tipo inquisitorio è più frequentemente associato alla figura del giudice-funzionario, la cui carriera ricalca del resto la struttura piramidale della giurisdizione, mentre quello accusatorio è più frequentemente amministrato da un giudice professionale inserito in una struttura in cui il coordinamento tende a prevalere sulla gerarchia (Merryman 1985; Cole, Frankowski et al. 1989; Delmas-Marty 1995), ciò che d'altronde può essere spiegato col fatto che simili costrutti presentano il vantaggio di una maggiore coerenza interna. Ed è per questo motivo che la partizione tra paesi di common law e di civil law rimane anche in questo caso il punto di partenza per considerare le principali coordinate dei rispettivi assetti processuali.

L'Inghilterra e gli Stati Uniti sono senza dubbio i paesi che più si avvicinano al processo di tipo adversary, ma l'adesione a questo modello è più accentuata nel settore penale che in quello civile.

Comunque, benché l'evoluzione riscontrata nei sistemi angloamericani mostri una crescita tendenziale dei poteri processuali del giudice - che è stata in certa misura sollecitata anche 
dalla diffusione degli studi comparati e dalla maggiore conoscenza degli istituti continentali (Frase 1990) - le differenze rispetto al panorama europeo rimangono tuttora significative. La collocazione istituzionale del giudice conserva qui le tracce lasciate dal progetto assolutista di centralizzazione del potere, che si realizza anche con la creazione di corti chiamate ad applicare le sole norme emanate dal principe e che gradualmente riescono a soppiantare le antiche giurisdizioni cetuali (Stein 1987, 15 ss.). La tradizione inquisitoria, imperniata sulla figura del giudice-funzionario, evolve quasi senza soluzioni di continuità dalla fine del medio evo sino ai nostri giorni. Sia pure lentamente, essa sviluppa tuttavia alcune garanzie fondamentali a tutela delle parti e dell'imputato, che costituiscono del resto un patrimonio ormai condiviso da tutte le democrazie contemporanee. Fra queste basterà ricordare il principio di presunzione di innocenza e più in generale il rafforzamento dei diritti della difesa nonché, per quanto attiene al contenzioso civile, il diritto riconosciuto ai litiganti di definire in piena autonomia le questioni da sottoporre al giudice. D'altra parte, la circolazione delle idee ha interessato anche l'Europa continentale, che ha a propria volta importato alcuni istituti processuali propri dei sistemi di common law. L'esempio più recente in questo senso è dato dall'Italia, che ha da poco adottato un nuovo codice di procedura penale a vocazione accusatoria. Ciò nonostante, il giudice assume in questo contesto un ruolo chiaramente più attivo. Le sue prerogative, particolarmente quelle di natura compensatoria nei confronti delle parti socialmente deboli, possono essere considerate come il prolungamento nel settore della giustizia dell'intervento pubblico che ha accompagnato la crescita dello stato sociale. Tali prerogative si manifestano attraverso i poteri di iniziativa, impulso e direzione che il giudice assume non soltanto nella fase dibattimentale ma anche, e soprattutto, nella fase preliminare, l'istruttoria. In breve, se quello anglosassone è un «processo degli avvocati» quello di civil law si profila piuttosto come un processo che trova nel giudice il proprio protagonista (Perrot 1993).

\section{Il controllo di costituzionalità delle leggi}

È difficile immaginare una prerogativa più penetrante di quella attribuita al giudice quando è chiamato a controllare la 
costituzionalità di una legge votata da una maggioranza politica, o comunque da un'istituzione che la rappresenta, e a prendere una decisione vincolante che può validarla o abrogarla. Non ci occuperemo in questa sede della vexata quaestio riguardante la legittimità democratica di tale funzione di controllo, che attiene essenzialmente al discorso prescrittivo (Troper 1990). Del resto, come Shapiro $(1990,335)$ ha osservato, gli studiosi generalmente concordano che «un poco» di controllo è necessario, ma quando si tratta di definire in concreto questo «poco» il dibattito resta aperto. Per quanto ci riguarda sarà sufficiente ricordare che la maggior parte delle democrazie contemporanee si è dotata di un controllo di costituzionalità delle leggi e che tutte hanno attribuito questo compito a strutture giurisdizionali. Le forme del controllo, la sua ampiezza e i modi con cui può essere attivato restano ciò nonostante diversi. $\mathrm{E}$ diverse sono anche le loro rispettive implicazioni quanto alla ripartizione delle competenze - e dunque di potere - tra giudici costituzionali e giudici ordinari, sempre che questi siano distinti, ciò che d'altra parte non sempre si verifica. Benché la distanza tra i sistemi di giustizia costituzionale tenda oggi a ridursi, almeno se si ha riguardo al ruolo che di fatto assumono nei regimi democratici, le condizioni proprie del periodo e dei contesti in cui sono stati introdotti hanno lasciato un'impronta tuttora visibile e concorrono a spiegare almeno in parte le numerose differenze che ancora sussistono (von Beyme 1992, 37).

L'introduzione e lo sviluppo del controllo di costituzionalità delle leggi sono stati molto più precoci e rapidi negli Stati Uniti. Pur senza attribuire esplicitamente tale funzione alla Corte suprema, il sistema americano presenta infatti sin dalla sua nascita nel 1787 le condizioni che preludono a questo esito: una costituzione scritta, concepita come legge superiore e dotata di un vero e proprio catalogo di diritti fondamentali ${ }^{10}$, la cui tutela pone altrettanti limiti all'esercizio del potere; un assetto federale, che esige la divisione del potere piuttosto che la sua concentrazione, e che affida al giudiziario il compito di dirimere i conflitti fra autorità centrali e locali; un'architettura costituzionale che riflette la diffidenza verso il primato delle assemblee legislative, quale fonte di una possibile «tirannia della maggioranza» a detrimento delle libertà individuali, e costruita sull'idea guida

10 In realtà il Bill of Rights è posteriore alla Costituzione federale, essendo stato adottato nel 1791 . 
che il potere deve essere limitato dal potere; un giudiziario concepito per affiancare e bilanciare, a parità di rango costituzionale, le altre due branche dello stato, il legislativo e l'esecutivo (Rebuffa 1993, 32-38). Considerata da questa angolazione, la sentenza con cui nel 1803 la Corte suprema si attribuisce il controllo di costituzionalità - trasformandosi in giudice della legge e non solo dei cittadini - non fa altro che portare a compimento l'assetto tracciato pochi anni prima e non suscita infatti reazioni apprezzabili da parte del sistema politico.

Lo sviluppo della giustizia costituzionale è invece più tardivo in Europa. Dopo l'esperienza dei parlamenti dell'ancien régime - le corti d'appello che rivendicarono il diritto di vigilare sul rispetto delle «leggi fondamentali della monarchia» (Cappelletti 1989, 124) - bisognerà attendere il XX secolo per assistere all'introduzione di un controllo di costituzionalità delle leggi, con la creazione nel 1920 della corte costituzionale austriaca. Nello stesso periodo anche la Repubblica di Weimar istituisce una controllo di conformità alla Legge fondamentale degli atti normativi (Schwietzke 1992, 152-154). Ma è solo dopo la seconda guerra mondiale che la giustizia costituzionale inizia la propria espansione sul continente. Dapprima la Germania con la Bundesverfassungsgericht, attivata nel 1951, seguita dall'Italia la cui Corte costituzionale, pur prevista dalla Carta del 1948, viene istituita solo nel 1956. Due anni dopo, la transizione alla V Repubblica sfocia anche in Francia nell'istituzione del Conseil Constitutionnel che, nonostante le sue molte peculiarità, acquista un peso crescente nel sistema politico francese. I paesi della penisola iberica si allineano al quadro europeo rispettivamente nel 1978, anno che segna la nascita del Tribunal constitucional in Spagna, e nel 1983 con l'attivazione dell'omonimo Tribunale portoghese. Infine, pur restando al di fuori del nostro campo d'analisi, anche i paesi dell'Europa centrale ed orientale seguono l'esempio delle democrazie occidentali dotandosi di corti costituzionali disegnate sulla falsariga del modello europeo (Schwarz 1992). Un simile ritardo in rapporto al sistema americano non dipende però solo dal più lento cammino verso la democrazia, benché sia evidente che questo rappresenta comunque un elemento importante. Può e deve essere messo in relazione anche con i modi di organizzare e distribuire il potere politico $o$, più precisamente, con gli assetti costituzionali che si affermano nell'Europa continentale sulla scia della rivoluzione francese. La centralità degli apparati legislativi, che con la 
progressiva estensione del suffragio elettorale si affermano come depositari via via più rappresentativi della volontà sovrana del popolo, ne è il tratto più qualificante e quello che meglio spiega la resistenza a qualsiasi forma di controllo esercitata da un'autorità esterna ed indipendente. Diversamente dal modello americano, questo assetto non tollera alcuna limitazione alla supremazia della maggioranza, né tanto meno accetta una legge superiore concepita come norma di diritto positivo e dunque tale da porre vincoli concreti alle espressioni della volontà maggioritaria. I testi costituzionali e gli stessi cataloghi dei diritti assumono in questo caso valore di semplici enunciati, certo importanti ma essenzialmente programmatici, e suscettibili pertanto di essere emendati in qualsiasi momento da un'assemblea che si pone ad un tempo come potere legislativo e costituente. Il connubio fra sovranità popolare e primato del parlamento rappresenta in altre parole un freno particolarmente efficace all'adozione di un controllo di costituzionalità esercitato secondo le forme e i modi della giurisdizione (von Beyme 1992). Non va però dimenticato che anche negli assetti europei esiste un potere da limitare, quello giudiziario. Il ricordo degli abusi perpetrati dai tribunali dell'ancien régime e, più in generale, il temuto insorgere di forme di «governo dei giudici» ${ }^{11}$ si traducono in un'architettura costituzionale fondata sulla separazione piuttosto che sul bilanciamento dei poteri. Il suo baricentro politico si situa sull'asse che va dal legislativo all'esecutivo ed esclude il giudiziario, cui si disconosce la qualità di terzo potere dello stato. Dunque, un'autorità giudiziaria, e non un potere giudiziario, collocata all'esterno dei circuiti politici. Da ultimo, anche l'organizzazione burocratica della magistratura è un elemento importante in questo quadro. Se da un lato essa pone di fatto dei vincoli gerarchici alla funzione giurisdizionale, dall'altro diventa un solido argomento per rifiutare al giudice-funzionario, legittimato sulla base della sola competenza tecnica e come tale politicamente non responsabile, un potere e delle prerogative dalle chiare implicazioni politiche ${ }^{12}$.

11 L'espressione, coniata da Boudin (1912), è stata introdotta in Francia da Lambert (1922). Sul punto si veda l'analisi di Davis (1987).

12 Cfr. Rebuffa (1993). Nel contesto europeo del secondo dopoguerra le corti costituzionali assumono inizialmente un ruolo per certi aspetti ambiguo. Esse sembrano nascere anche dalla diffidenza nei confronti della giurisdizione ordinaria e con l'intento di controllarla (Cappelletti 1989), ma si tratta di una tendenza più accentuata nell'Europa del Sud. 
Nonostante il loro compito principale consista nel sindacare la conformità delle leggi alla costituzione, le Corti costituzionali hanno spesso altre e non meno delicate funzioni. Sono infatti chiamate a risolvere i conflitti fra i poteri dello stato o anche tra governo centrale e governo locale, vigilando in tal modo sul rispetto dei confini imposti alle rispettive competenze, e a proteggere i diritti individuali dai possibili abusi da parte delle autorità pubbliche (Marradi 1972, 522). Stante l'importanza delle loro attribuzioni, si ritiene generalmente che le corti, ancorché qualificate come organi propriamente giurisdizionali, assumano in pratica un ruolo ibrido, a metà strada fra giustizia e politi$\mathrm{Ca}^{13}$. Il dualismo di questa loro posizione istituzionale, e particolarmente il suo versante politico, è del resto apertamente riconosciuto persino nei paesi ove la concezione esecutoria del ruolo giudiziario vanta una lunga tradizione, come ad esempio in Germania (Teubner 1984), e diviene evidente quando si passa a considerare la struttura di queste corti. Se per un verso sono concepite come contrappeso agli altri poteri, così da temperare il principio maggioritario che li guida, dall'altro sono comunque questi stessi poteri a definirne la composizione. Detto altrimenti, i controllori sono scelti dai controllati (von Brünneck 1992b, 224). Il paradosso è del resto solo apparente. Una soluzione di questo tipo risponde infatti al principio di bilanciamento dei poteri - che in misura maggiore o minore informa le democrazie costituzionali - ed ha inoltre il vantaggio di conferire alle corti una sorta di legittimazione politica a fronte del ruolo esso stesso politico che di fatto assumono (Dahl 1957; Cappelletti 1981b, 641-642). I giudici costituzionali sono dunque nominati dalle altre branche dello stato: l'esecutivo e il legislativo, più raramente il giudiziario. Sotto questo profilo conviene distinguere $i$ sistemi in cui ciascun potere provvede separatamente alla designazione dei giudici costituzionali da quelli che si affidano invece alla collaborazione tra i diversi apparati coinvolti nel processo di scelta (vedi tab. 2).

Quest'ultimo caso è ben rappresentato dagli Stati Uniti. I nove giudici della Corte suprema sono prescelti seguendo la stessa procedura che regola il reclutamento dei loro colleghi federali e che gravita attorno alla complessa opera di mediazione

13 Così Mény (1990, 299), ma già Ancel (1934) aveva sottolineato che l'attribuzione di tali funzioni alle corti supreme non può che esaltare il carattere politico del loro ruolo. 


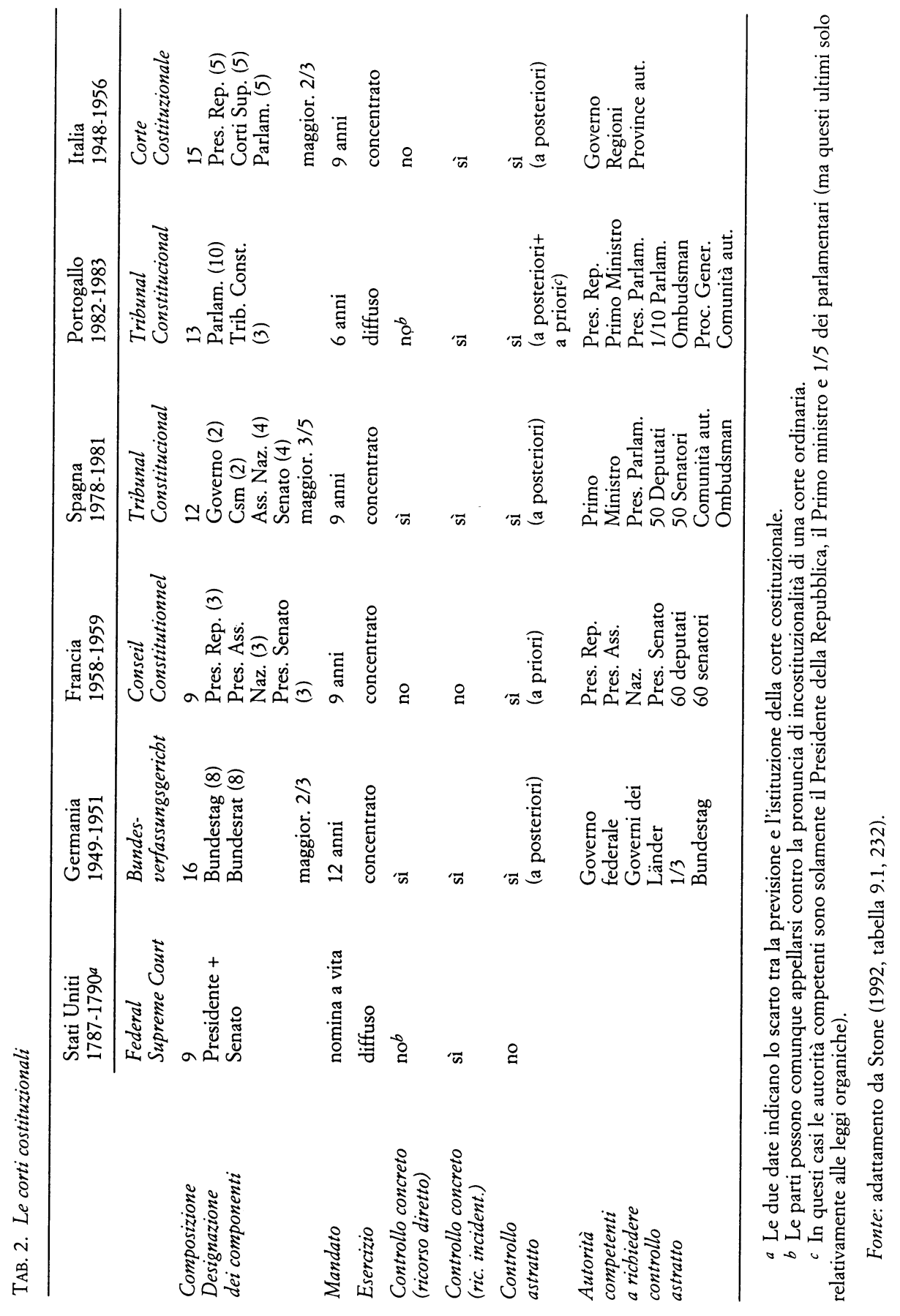


fra due attori principali: il Presidente e il Senato. La prima soluzione - che annovera, fra gli altri casi, la Germania, l'Italia, la Spagna e la Francia - può invece essere letta più agevolmente nell'ambito della dottrina di separazione dei poteri che, così come tradizionalmente intesa nell'Europa continentale, esprime una diffidenza di fondo verso ogni commistione fra i diversi apparati potestativi (Rebuffa 1993). Va però tenuto presente che i partiti politici sembrano ovunque esercitare un'influenza piuttosto elevata sulla scelta dei giudici costituzionali (von Brünneck 1992b). Non è quindi da escludere la possibilità che i partiti rappresentino dei canali di comunicazione capaci di coordinare, almeno in certa misura, apparati che operano in modo formalmente autonomo l'uno dall'altro. Per quanto attiene al ruolo giocato dalle assemblee legislative, la «collaborazione» tra le forze politiche presenti in parlamento può dipendere sia da prassi consolidate, come ad esempio si verifica in Austria, sia dal tipo di maggioranza richiesta per eleggere i giudici. In Germania, ad esempio, le procedure di designazione sembrano sinora aver assicurato una composizione della Corte sufficientemente equilibrata, che ha sempre visto la presenza di componenti indicati dall'opposizione (Landfried 1992; Schwietzke 1992, 162). Nel caso dell'Italia l'influenza dei partiti politici è esplicita per quanto attiene alla quota di giudici eletti dal Parlamento - secondo criteri che tengono conto del peso specifico dei gruppi principali, ivi inclusa l'opposizione - ma anche le designazioni presidenziali sembrano risentire dello stesso tipo di logica (Pizzorusso 1992, 111). Pur trattandosi di un fenomeno oltremodo accentuato in Italia, in ragione della tradizionale predominanza dei partiti, la spinta «proporzionalista» resta comunque una dato comune anche ad altri paesi, almeno in occasione dell'elezione dei giudici costituzionali (von Brünneck 1992a, 32-34).

La struttura delle Corti costituzionali offre un primo elemento utile ad inquadrare l'assetto istituzionale dei rapporti tra giustizia e politica, ma tale relazione può essere meglio apprezzata passando a considerare la collocazione delle corti nel sistema giudiziario, i modi di esercizio delle relative funzioni e le loro implicazioni più rilevanti (Favoreu e Jolowicz 1984; Jackson e Tate 1992). Un primo elemento concerne dunque il locus istituzionale del sindacato di costituzionalità delle leggi. La partizione classica al riguardo distingue tra un controllo «concentrato» opposto ad uno «diffuso» (Cappelletti 1971; Favoreu 1981). Mentre nel primo caso tale funzione viene demandata ad 
una corte ad boc, nel secondo essa si presenta distribuita lungo l'intera piramide giurisdizionale, con la conseguenza che ogni giudice in qualunque grado di giudizio può rifiutare di applicare una norma ritenuta contraria alla costituzione. Gli archetipi di questi due modelli sono rappresentati rispettivamente dalla corte austriaca del 1920 , secondo il disegno tracciato da Hans Kelsen, e quello americano inaugurato dalla sentenza Marbury v. Madison. Negli Stati Uniti il controllo di costituzionalità viene infatti esercitato a tutti i livelli della giurisdizione e si traduce nel potere di ciascun giudice di disapplicare una norma relativamente al solo caso in esame, ciò che dunque non implica l'abrogazione della norma così censurata. Il rilievo del ruolo giocato in questo senso dalla Corte suprema, e dunque la ragione per cui l'abbiamo sinora assimilata alle altre corti europee, risiede nella dottrina dello stare decisis, grazie alla quale le decisioni rese dalle corti superiori vincolano quelle inferiori. $\mathrm{Ne}$ consegue che solo la censura pronunciata al vertice della piramide giurisdizionale equivale ad una dichiarazione di nullità con effetto abrogativo. Mentre questo modello contraddistingue le ex colonie del Regno Unito - che tuttavia non conosce alcun controllo di costituzionalità, sia per la mancanza di una legge fondamentale in forma scritta sia, e soprattutto, per il primato riconosciuto al parlamento - il secondo ha invece segnato l'espansione della giustizia costituzionale in Europa, ma va aggiunto che tanto la Svezia quanto il Portogallo conoscono forme, sia pure diverse, di controllo diffuso (von Brünneck 1992a; Holmström 1995, 358). Il quadro sin qui tracciato riflette dunque in buona sostanza lo spartiacque tra le due tradizioni giuridiche di common law e civil law. Quest'ultima denota ancora una volta la sua propensione a frammentare la giurisdizione, sottraendo così al giudice ordinario un contenzioso di indubbio rilievo politico. Le implicazioni di questi due diversi assetti sull'ampiezza dei poteri della magistratura sono dunque evidenti, ma $\mathrm{i}$ giudici continentali conservano comunque alcune importanti prerogative che, senza porli sullo stesso piano dei loro omologhi americani, non sono tuttavia da sottovalutare.

Un secondo aspetto da tener presente riguarda invece il momento in cui il giudice costituzionale può essere attivato. Contrariamente a quanto si potrebbe forse ritenere, la questione non è affatto secondaria. Si tratta invece di un buon indicatore circa la posizione riservata al legislativo nell'assetto costituzionale. Non ci sono che due alternative: l'intervento della corte 
può essere sollecitato prima o dopo l'entrata in vigore di una legge. Il controllo detto «a priori» caratterizza il Consiglio costituzionale francese, che può infatti essere adito sono nel breve intervallo di tempo che intercorre fra l'emanazione di una legge e la sua promulgazione. Una volta promulgata, la legge diviene cioè inattaccabile e può essere emendata o abrogata solamente dal legislatore, che conserva in tal modo il tradizionale primato sul processo politico. Di converso, il controllo «a posteriori», che ritroviamo in tutti gli altri casi, consente di invocare l'intervento del giudice costituzionale in qualsiasi momento al fine di tutelare diritti che si ritengono violati dalla norma. Detto altrimenti, la volontà espressa da una maggioranza politica può in qualsiasi momento essere sfidata da un'azione che, nella maggior parte dei casi, è promossa su base individuale.

Poiché la passività è una «virtù» per lo più condivisa anche dalle corti costituzionali - la sola parziale eccezione al riguardo è data dal potere della Corte suprema americana di scegliere $\mathrm{i}$ casi - è importante precisare quali sono i soggetti che possono azionarle e quali sono gli strumenti di cui dispongono per farlo. Un primo canale di accesso, che assicura il massimo grado di apertura del sistema, è quello che consente a qualsiasi cittadino di adire il giudice costituzionale in presenza di una presunta violazione dei diritti fondamentali, diritti cioè esplicitamente enumerati dalla costituzione stessa, da parte di un atto normativo o anche di un atto posto in essere da una qualsiasi autorità pubblica. È questo il caso del recurso de amparo, previsto dall'art. 161 della costituzione spagnola e che rappresenta il $90 \%$ circa del carico di lavoro del Tribunale costituzionale (Stone 1992, 233). Il ricorso diretto è stato introdotto anche in Germania sin dal 1969 e rappresenta addirittura il 95\% circa del contenzioso annualmente trattato dalla Corte costituzionale (Landfried 1992, 152-153).

Una seconda via d'accesso è ugualmente aperta a tutti i cittadini, ma solo a condizione che siano parte in causa in un processo. Attraverso il ricorso cosiddetto «incidentale», i litiganti hanno cioè la possibilità di sollevare una questione di legittimità costituzionale, che dovrà peraltro essere circoscritta alle sole norme ritenute applicabili nel procedimento che li vede coinvolti. Spetta poi al giudice valutare la fondatezza della questione e rimetterla eventualmente alla Corte costituzionale. Non meno importante è il fatto che, a prescindere da qualsiasi iniziativa di parte, il giudice stesso può eccepire la costituzionalità di 
una norma applicabile al caso in esame. Il giudice assume dunque il ruolo di filtro necessario tra i singoli e la Corte costituzionale, che non può essere attivata se non sulla base di una sua decisione motivata. Stante questa procedura, non va trascurata la possibilità che il ricorso incidentale diventi un importante veicolo di «politica giurisprudenziale», uno strumento cioè cui il giudice può ricorrere per cercare di affermare orientamenti personali o anche condivisi dal gruppo di riferimento, senza dimenticare che, per quanto attiene alle magistrature burocratiche, quest'ultimo è spesso rappresentato da una associazione di categoria o da una corrente al suo interno ${ }^{14}$. In ogni caso, in virtù di questa procedura il giudice ordinario diventa un segmento obbligato della funzione di controllo giurisdizionale delle leggi, con la conseguenza di bilanciare almeno in parte la concentrazione del relativo potere nelle mani di una corte speciale.

Il ricorso incidentale dà luogo a ciò che si suole definire un controllo «concreto» delle leggi, perché innescato dall'applicazione di una norma ad un caso specifico. Ritroviamo questo tipo di controllo nella maggior parte dei paesi europei sin qui menzionati - Germania, Spagna e Italia - che d'altronde riconoscono il potere di attivare le rispettive corti costituzionali non solo alle giurisdizioni ordinarie ma anche a quelle speciali o specializzate (amministrative, del lavoro, ecc.). Rispetto a questo quadro l'eccezione è rappresentata dal Consiglio costituzionale francese. Questi esercita le proprie funzioni rispetto a norme che, non essendo ancora promulgate, non possono essere oggetto di alcuna applicazione, giurisdizionale o meno. L'azione del Consiglio è dunque circoscritta al cosiddetto controllo «astratto», una competenza condivisa peraltro con le altre Corti costituzionali europee, anche se con modalità di volta in volta diverse. Dato comune è che in tutti i casi tale controllo può essere attivato solo dai poteri dello stato, ad esclusione del giudiziario, o comunque da autorità pubbliche: l'esecutivo, il legislativo, i governi locali o anche, il defensor del pueblo, ossia la versione

14 Un esempio in questo senso è dato effettivamente dal caso italiano ove, a partire dalla seconda metà degli anni ' 60 , Magistratura Democratica elabora un'ideologia professionale che vede nella costituzione il principale referente della funzione giudiziaria e sollecita l'applicazione diretta delle norme costituzionali, ove possibile, nonché un uso intensivo del ricorso incidentale ogniqualvolta il giudice creda di ravvisare una difformità tra il dettato costituzionale e le leggi ordinarie. Per una recente ricostruzione di tale orientamento si veda Ferrajoli (1994) e, per un bilancio critico, Guarnieri (1992, 120-122). 
spagnola dell'Ombudsman. Contrariamente a quanto si potrebbe immaginare, il controllo astratto ha dato prova, particolarmente in Francia, di un potenziale che sembra aver superato persino le intenzioni dei suoi stessi fautori e che è ben documentato proprio dall'evoluzione che ha segnato la storia recente del Consiglio. All'atto della sua istituzione, il Consiglio poteva essere attivato da sole quattro autorità, coincidenti del resto con altrettante persone fisiche: il Capo dello stato, il Primo ministro, il Presidente del Senato e quello dell'Assemblea nazionale. Dopo la «miniriforma» del 1974 , che ha attribuito tale potere a 60 deputati o senatori, fissando così una soglia accessibile anche all'opposizione, si è assistito ad un notevole incremento dei ricorsi, che fino a quella data assommavano a poche unità (Stone 1992). Il controllo astratto viene cioè utilizzato come strumento per trasferire, e in un certo senso per prolungare, $\mathrm{i}$ conflitti politici dall'arena parlamentare a quella giurisdizionale, che tende in tal modo ad assumere il ruolo di una «terza camera legiferante» (Shapiro 1990, 541). L'inserimento nel processo legislativo di un nuovo attore, che diversamente dai suoi interlocutori istituzionali ha però l'ultima parola sulle sorti di una legge, sembra inoltre avere in parte modificato i rapporti fra $i$ gruppi parlamentari e dunque le dinamiche del dibattito politico. La semplice eventualità, quando non la minaccia, di adire il Consiglio, se da un lato spinge la maggioranza ad elaborare i testi normativi con una maggiore attenzione ai loro risvolti giuridici, in modo da prevenire possibili censure di costituzionalità, dall'altro apre alle opposizioni margini di manovra che le erano preclusi fino al '74. Con la conseguenza che, mentre il discorso politico tende a mutuare il linguaggio del diritto, quello giuridico sembra essere divenuto molto più sensibile alle valutazioni propriamente politiche (Stone 1994). L'esperienza francese non è facilmente generalizzabile, non foss'altro perché negli altri paesi i ricorsi di questo tipo rappresentano una parte decisamente esigua dei carichi di lavoro delle altre corti costituzionali, ma anche la Germania sembra denotare un'evoluzione per certi aspetti simile a questa.

I giudici continentali partecipano dunque al controllo di costituzionalità in maniera comparativamente limitata rispetto a quelli americani, essendo tale funzione sostanzialmente monopolizzata da apposite corti. Tuttavia la creazione e la progressiva espansione di sistemi di giustizia sovranazionale, come quelli istituiti dalla Comunità europea o dalla Convenzione dei 
diritti dell'uomo, hanno aperto nuovi spazi di intervento che ampliano le competenze e i margini di apprezzamento discrezionale della magistratura ordinaria, posto beninteso che essa intenda riempirli, anche se è difficile immaginare che la tendenza in questo senso possa essere invertita. Il diritto comunitario si impone ormai come un vero e proprio ordine giuridico sovranazionale dotato, se non di una costituzione in senso stretto, ossia un documento unitario in forma scritta, quanto meno di un insieme di regole di diritto positivo e relativamente organiche, che pongono dei limiti alla sovranità degli stati membri imponendosi sui singoli sistemi giuridici nazionali. Una parte consistente delle norme che disciplinano i rapporti tra gli stati membri sono state elaborate dalla Corte europea di giustizia, che rappresenta un esempio emblematico di interventismo giudiziario. E stata infatti la Corte del Lussemburgo a dichiarare che compete ai giudici nazionali far valere ed applicare direttamente il diritto comunitario nei rispettivi sistemi. Ciò implica una valutazione di conformità della legislazione nazionale a quella comunitaria e dunque il potere del singolo giudice di disapplicare la prima in favore della seconda. In altre parole, si tratta di una sorta di controllo costituzionale esercitato in maniera diffusa, fatto questo che si pone come un elemento del tutto nuovo nel panorama europeo (Cappelletti 1994, 188). La Corte di giustizia resta comunque la chiave di volta del sistema. Attraverso il cosiddetto rinvio pregiudiziale, qualsiasi giudice nazionale può sospendere un processo in corso di svolgimento per chiedere alla Corte una pronuncia relativa sia alla validità sia all'interpretazione di una norma comunitaria ritenuta applicabile in tale processo. Non è a caso, del resto, che gli sviluppi più interessanti in questo senso siano stati osservati proprio in Inghilterra che, come si è detto, non conosce alcun controllo di costituzionalità delle leggi. In forza di tali sviluppi, anche $\mathrm{i}$ giudici inglesi possono ora disapplicare una legge nazionale ritenuta in contrasto con una disposizione comunitaria. Ciò sembra in effetti aver sollecitato un maggior attivismo da parte loro, documentato dall'incremento osservato nei ricorsi alla Corte del Lussemburgo a partire all'incirca dalla seconda metà degli anni '80 (Norton 1991, 493; Sunkin 1995). 


\section{Conclusioni}

Sulla base del quadro che abbiamo sin qui ricostruito, è ora possibile enucleare i caratteri relativi all'assetto del sistema giudiziario che, a prescindere dagli altri elementi che pure entrano in gioco, sembrano favorire una crescita del suo rilievo politico.

Come si è visto, un primo insieme di elementi attiene alla struttura del sistema. In questo ambito la prima dimensione da considerare è data dall'area d'intervento del giudice, ossia dal tipo di casi che questi può decidere. Quanto più ampia è l'estensione delle sue competenze, tanto maggiore sarà l'incidenza politica del suo ruolo. In linea di principio ogni forma di frammentazione della giurisdizione comprime infatti l'impatto potenziale della giustizia. Al verificarsi di questa ipotesi, è però necessario considerare la collocazione delle corti speciali nell'ambito del sistema, l'esistenza o meno di un vertice a giurisdizione unificata, nonché le eventuali connessioni tra i giudici che le formano e il sistema politico. Come si è visto, il grado di frammentazione è certamente più elevato nell'Europa continentale che, con la sola eccezione della Spagna, si caratterizza per la netta cesura tra corti ordinarie ed amministrative; una cesura che a propria volta si riflette nel diverso status dei giudici che le formano, le cui garanzie istituzionali di indipendenza sono più solide nel primo caso $\mathrm{e}$ tendenzialmente più attenuate nel secondo. Più ambiguo è invece il ruolo giocato dalla seconda dimensione individuata, che riguarda la coerenza giurisprudenziale e il peso specifico delle Corti supreme nelle dinamiche interne al sistema giudiziario. La presenza di un vertice decisionale forte e in grado di catalizzare una porzione consistente dei flussi decisionali, se da un lato aumenta la coerenza del sistema e dunque la sua incidenza politica, dall'altro riduce l'autonomia interna dei livelli inferiori della piramide; autonomia che costituisce invece una condizione necessaria per l'instaurarsi di rapporti più stretti, e verosimilmente anche più frequenti, tra il sistema giudiziario e il suo ambiente politico e sociale. Sotto questo profilo è evidente che i sistemi continentali denotano un grado di centralizzazione più elevato di quelli anglosassoni. D'altra parte, l'allentarsi dei vincoli organizzativi che tradizionalmente caratterizzavano le magistrature burocratiche, in particolare la carriera, si accompagna ad un indebolimento dell'autorità «didattica» delle corti supreme e dunque della loro centralità, fatto questo ormai evidente in Italia ma che si sta in certa misura manifestando anche in Francia. 
L'ampiezza della giurisdizione è però condizione necessaria ma non sufficiente per avere una giustizia politicamente incisiva. Il secondo blocco di elementi da considerare riguarda infatti i meccanismi che regolano l'accesso al sistema e le concrete opportunità offerte ai soggetti individuali e collettivi di invocare l'intervento del giudice. Quanto più basse sono le soglie di accesso al sistema, particolarmente per i gruppi di interesse, tanto più importante sarà il peso relativo della giustizia. Posto che altre condizioni risultino soddisfatte, questa potrà infatti essere utilizzata come canale di articolazione delle domande politiche a fianco, e in alternativa, agli altri canali istituzionali. Sotto questo profilo i paesi dell'Europa continentale presi in esame stanno recuperando parte dello scarto che tradizionalmente li separava dagli Stati Uniti, grazie alla progressiva diffusione di forme di rappresentanza in giudizio che aprono il sistema a gruppi di interesse in precedenza esclusi dall'arena giudiziaria. Non meno importante, e talvolta cruciale, è il ruolo svolto dal pubblico ministero nella sua qualità di gatekeeper, il guardiano dei cancelli, nel settore penale. L'analisi comparata indica che un pubblico ministero indipendente e nel contempo legato al corpo giudiziario sul piano organizzativo e culturale - il caso cioè delle strutture a corpo unico - è un elemento che esalta fortemente il rilievo politico della magistratura.

Infine, i poteri del giudice. In questo caso è evidente che, ceteris paribus, un assetto processuale di tipo inquisitorio, che demanda al giudice un ruolo più attivo, tende ad accrescerne la capacità di intervento sull'ambiente politico e sociale. Questa stessa osservazione vale egualmente per il controllo di costituzionalità, nella misura in cui la magistratura ordinaria partecipa a tale funzione. Come si è visto, diversamente dagli Stati Uniti, il modello europeo concentra tale potere nelle mani di apposite corti, ma lascia ai giudici ordinari il compito di filtrare i ricorsi, ciò che ne aumenta in modo sensibile i margini di intervento nonché di apprezzamento discrezionale. È il principio di subordinazione alla legge, tradizionalmente molto forte nell'Europa continentale, a risultarne in certa misura allentato. Le prerogative in tal modo assunte dalle magistrature europee vengono d'altronde ulteriormente rafforzate dagli sviluppi in atto sul piano comunitario. Il controllo diffuso delle leggi, che il legislatore continentale ha respinto all'interno dei singoli ordinamenti nazionali, ricompare sulla scena sovranazionale traducendosi, lo abbiamo appena visto, nel potere formalmente riconosciuto a 
tutti i giudici di disapplicare una legge nazionale ritenuta in contrasto con una norma comunitaria.

\section{Riferimenti bibliografici}

Ancel, M. (1934), Réflexions sur l'étude comparative des Cours Suprêmes et le «recours en Cassation», in «Annales de l'Institut de Droit Comparé de l'Université de Paris», pp. 285-302.

Becker, T. (1970), Comparative Judicial Politics, Chicago, Rand McNally.

Blankenburg, E. (1994), La mobilisation du droit. Les conditions du recours et du non-recours à la Justice, in «Droit et Societé», XXVIII, pp. 691-703.

Blankenburg, E. e J. Cooper (1982), A Survey of Literature on Legal Aid in Europe, in «The Windsor Yearbook of Access to Justice», 2, pp. 263-292.

Boudin, L.B. (1912), Government by Judiciary, in «Political Science Quarterly», XXVI, pp. 238-270.

Calamandrei, P. (1965), Processo e democrazia, in Opere giuridiche, Napoli, Morano, vol. I, pp. 76-112.

Cappelletti, M. (1981a) (a cura di), Acces to Justice and the Welfare State, Firenze, Le Monnier.

Cappelletti, M. (1969), Processo e ideologie, Bologna, Il Mulino.

- (1971), Judicial Review in the Contemporary World, Indianapolis, Bobbs-Merrill.

- (1981b), Nécessité et légitimité de la justice constitutionnelle, in «Revue Internationale de Droit Comparé», XXXIII, pp. 625-657.

- (1989), The Judicial Process in Comparative Perspective, Oxford, Clarendon Press.

- (1994), Le dimensioni della giustizia, Bologna, Il Mulino.

Chayes, A. (1976), The Role of the Judge in the Public Law Litigation, in «Harvard Law Review», LXXXIX, pp. 1281-1316.

Cole, G.F., S.J. Frankowski e M.G. Gertz (1989), Major Criminal Justice Systems, Newbury Park, Sage (II ed.).

Crawford, A. (1994), L'accès à la justice et ses alternatives en Angleterre et au Pays de Galles, relazione presentata al colloquio «Justice et Société», Lille, Enm-Ihej.

Dahl, R.A. (1957), Decision-Making in a Democracy: the Supreme Court as a National Policy-Maker, in «The Journal of Public Law», 6, pp. 279-295.

Damaska, M.R. (1975), Structure of Authority and Comparative Criminal Procedure, in «The Yale Law Journal», LXXXIV, pp. 480545.

- (1991), I volti della giustizia e del potere, Bologna, Il Mulino.

Davis, M.H. (1987), A Government of Judges: An Historical View, in 
«The American Journal of Comparative Law», XXXV, pp. 559-580.

Delmas-Marty, M. (a cura di) (1995), Procédures pénales d'Europe, $\mathrm{Pa}$ ris, PUF.

Díez-Picazo, L.M. (1995), Il problema della discrezionalità nell'esercizio dell'azione penale, in «Rivista Trimestrale di Diritto Pubblico», 4, pp. 919-940.

Di Federico, G. (1995), Il pubblico ministero: indipendenza, responsabilità, carriera «separata», in «L'indice penale», XXIX, pp. 399 437.

Eckhoff, T. (1965), Impartiality, Separation of Powers, and Judicial Independence, in «Scandinavian Studies in Law», IX, pp. 11-48.

- (1976), The Relationships between Judicial and Political Branches of Government, in Friedman e Rehbinder (1976), pp. 14-23.

Fanchiotti, V. (1993), voce Sistema accusatorio e sistema inquisitorio, Roma, Enciclopedia Giuridica Treccani, vol. XXIX.

Favoreu, L. (a cura di) (1981), Cours constitutionnelles européennes et droits fondamentaux, Paris-Aix en Marseille, Economica-Presses Universitaires d'Aix en Marseille.

Favoreu, L. e J.-A. Jolowicz (a cura di) (1984), Le contrôle juridictionnel des lois, Paris-Aix en Marseille, Economica-Presses Universitaires d'Aix en Marseille.

Feeley, M.M. e M. Lazarson (1983), Police-Prosecution Relationships: An Interorganizational Perspective, in K.D. Boyum e L. Mather (a cura di), Empirical Theories about Courts, London, Longman, pp. 216-243.

Feldman, D. (1992), Civil Liberties and Human Rights in England and Wales, Oxford, Clarendon Press.

Ferrajoli, L. (1994), Per una storia delle idee di magistratura democrati$c a$, in N. Rossi (a cura di), Giudici e democrazia. La magistratura progressista nel mutamento istituzionale, Milano, Franco Angeli, pp. 55-79.

Frase, R.S. (1990), Comparative Criminal Justice as a Guide to American Law Reform, in «California Law Review», 78, pp. 549-683.

Friedman, L.M. (1976), Trial Courts and Their Work in the Modern World, in Friedman e Rehbinder (1976), pp. 25-38.

- (1994), Total Justice, New York, Russel Sage (II ed.).

Friedman, L.M. e M. Rhebinder (a cura di) (1976), Zur Soziologie des Gerichtsverfabrens, Opladen, Westdeutscher Verlag.

Friedrich, C.J. (1950), Constitutional Government and Democracy, Boston, Ginn \& Co.

Garapon, A. (1996), Le gardien des promesses, Paris, Odile Jacob.

Goldstein, A.S. e M. Marcus (1977), The Mith of Judicial Supervision in Three «Inquisitorial» Systems: France, Italy and Germany, in «The Yale Law Journal», 87, pp. 240-283.

Grossman, J.B. e A. Sarat (1981), Access to Justice and the Limits of 
the Law, in R.L. Gambitta, M.L. May e J.C. Foster (a cura di), Governing through Courts, Beverly Hills, Sage, pp. 77-92.

Guarnieri, C. (1984), Pubblico Ministero e sistema politico, Padova, CEDAM.

- (1992), Magistratura e politica in Italia, Bologna, Il Mulino.

Guarnieri, C. e P. Pederzoli (1996), La puissance de juger, Paris, Michalon.

Habib, L. (1992), Le Conseil Constitutionnel, in I.I. Kavass (a cura di), Supranational and Constitutional Courts in Europe: Functions and Sources, Buffalo-New York, Hein \& Co., pp. 215-245.

Haenel, H. e J. Harthuis (1991), Justice sinistrée: démocratie en danger, Paris, Economica.

Hazard, G.C. e M. Taruffo (1993), La giustizia civile negli Stati Uniti, Bologna, Il Mulino.

Holmström, B. (1995), Sweden, in Tate e Vallinder (1995), pp. 345 367.

Ingman, T. (1983), The English Legal Process, London, Financial Training.

Jackson, D.W. e C.N. Tate (1992), Comparative Judicial Review and Public Policy, Westport, Greenwood.

Lambert, E. (1922), Le gouvernement des juges et la lutte contre la legislation sociale aux Etats-Unis, Paris, Giard.

Landfried, C. (a cura di) (1992), Constitutional Review and Legislation. An International Comparison, Baden-Baden, Nomos.

Leigh, L.H. e L. Zedner (1992), A Report on the Administration of Criminal Justice in the Pre-trial Phase in France and Germany, London, HMSO.

Lindblom, H. e G.D. Watson (1991), Courts and Lawyers Facing Complex Litigation Problems, in Roles and Organization of Judges and Lawyers in Contemporary Societies. General Reports, IXth World Conference of Procedural Law, Coimbra-Lisboa, vol. I, pp. $540-600$.

Maillard de, J. (1994), Crimes et lois, Paris, Flammarion.

Majone, G. (1994), Independence vs. Accountability? Non-Majoritarian Institutions and Democratic Governement, Florence, European University Institute.

Marradi, A. (1972), Funzioni delle corti costituzionali nel sistema politico, in «Rivista Italiana di Scienza Politica», vol. 2, pp. 483-523.

Marshall, T.H. (1976), Cittadinanza e classe sociale, Torino, UTET.

Mény, I. (1990), Governement and Politics in Western Europe, Oxford, Oxford University Press.

Merryman, J.H. (1985), The Civil Law Tradition, Stanford, Stanford University Press (II ed.).

Norton, P. (1991), The Judiciary, in B. Jones (a cura di), Politics UK, London, Allen, pp. 475-498. 
Pederzoli, P. (1990), Il giudice nei regimi democratici, in «Rivista Italiana di Scienza Politica», vol. 20, 2, 293-323.

Peltason, J.W. (1968), voce Judicial Process, in International Encyclopedia of Social Sciences, New York, Mcmillan, VIII, pp. 283-291.

Perrot (1993), Institutions judiciaires, Paris, Montchretien.

Pizzorusso, A. (1992), Constitutional Review and Legislation in Italy, in Landfried (1992), pp. 109-126.

Rebuffa, G. (1993), La funzione giudiziaria, Torino, Giappichelli.

Sarat, A. e J.B. Grossman (1975), Courts and Conflict Resolution: problems in the Mobilization of Adjudication, in «American Political Science Review», LXIX, pp. 1200-1217.

Schwartz, H. (1992), The New East European Constitutional Courts, in «Michigan Journal of International Law», 13, pp. 741-785.

Schwietzke, J. (1992), Constitutional Courts of Germany, Austria and Switzerland, in I.I. Kavass (a cura di), Supranational and Constitutional Courts in Europe: Functions and Sources, Buffalo-New York, Hein \& Co., pp. 149-209.

Shapiro, M. (1980), Appeals, in «Law and Society Review», XIV, pp. 629-662.

- (1981), Courts. A Comparative and Political Analysis, Chicago, The University of Chicago Press.

- (1990), Judicial Review in France, in «Journal of Law and Politics», VI, pp. 531-548.

Stein, P. (1987), I fondamenti del diritto europeo. Profili sostanziali e processuali dell'evoluzione dei sistemi giuridici, Milano, Giuffrè.

Stone, A. (1992), The Birth of Judicial Politics in France, New York e Oxford, Oxford University Press.

- (1994), Judging Socialist Reform. The Politics of Coordinate Construction in France and Germany, in «Comparative Political Studies», 4, pp. 443-469.

Sunkin, M. (1995), The United Kingdom, in Tate e Vallinder (1995), pp. 67-78.

Tarello, G. (1988), Cultura giuridica e politica del diritto, Bologna, Il Mulino.

Taruffo, M. (1991), Il vertice ambiguo, Bologna, Il Mulino.

Tate, C.N. e T. Vallinder (a cura di) (1995), The Global Expansion of Judicial Power, New York, New York University Press.

Teubner, E. (1984), Die Bestellung zum Berufsrichter in Bund und Länder, Koeln, Carl Heymann.

Toharia, J.J. (1975), Judicial Independence in an Autboritarian Regime: The Case of Contemporary Spain, in «Law and Society Review», IV, pp. 475-496.

- (1976), Economic Development and Litigation: the Case of Spain, in Friedman e Rehbinder (1976), pp. 39-82.

Troper, M. (1990), Justice constitutionelle et démocratie, «Revue Francaise de Droit Constitutionnel», 1, pp. 31-48. 
Vallinder, T. (1995), When the Courts Go Marching In, in Tate e Vallinder (1995), pp. 13-26.

von Beyme, K. (1992), The Genesis of Constitutional Review in Parliamentary Systems, in Landfried (1992), pp. 21-38.

von Brünneck, A. (1992a), Verfassungsgerichtsbarkeit in den westlichen Demokratien. Ein systematischer Verfassungsvergleich, BadenBaden, Nomos.

- (1992b), Constitutional Review and Legislation in Western Democracies, in Landfried (1992), pp. 219-260.

Waltman, J.L. (1988), The Courts and the Political Change in Post-industrial Society, in Waltman e Holland (1988), pp. 216-234.

Waltman, J.L. e K. Holland (1988), The Political Role of Law Courts in Modern Democracies, London, Mcmillan.

Weigend, T. (1983a), Criminal Procedure: Comparative Aspects, in S. Kadish (a cura di), Encyclopedia of Crime and Justice, New York, The Free Press, vol. II, pp. 537-546.

- (1983b), Prosecution: Comparative Aspects, in S. Kadish (a cura di), Encyclopedia of Crime and Justice, New York, The Free Press, vol. II, pp. 1296-1304.

Weiler, P. (1968), Two Models of Judicial Decision-making, in «Canadian Bar Review», XLVI, 1968, pp. 406-471.

Zemans, F.K. (1983), Legal Mobilization: The Neglected Role of the Law in the Political System, in «American Political Science Review», LXXVIII, pp. 690-703. 